\title{
The Relativistic Jet-Accretion Flow-Wind Connection in Mrk 231
}

\author{
Cormac Reynolds ${ }^{1}$, Brian Punsly ${ }^{2}$, Giovanni Miniutti ${ }^{3}$, Christopher P. O’Dea ${ }^{4,5}$, Natasha \\ Hurley-Walker 6
}

\begin{abstract}
Long term radio monitoring of the broad absorption line quasar, Mrk 231, at 17.6 GHz detected a strong flare in 2015. This triggered four epochs of Very Long Baseline Array (VLBA) observations from $8.4 \mathrm{GHz}$ to $43 \mathrm{GHz}$ as well as three epochs of X-ray observations with NuSTAR and two with XMM over a 15 week period. Two ejected components were detected by the VLBA observations. A conservative lower bound on the apparent speed of the first ejection is attained by assuming that it was ejected when the flare began, $v_{\text {app }}>3.15 \mathrm{c}$. Serendipitous far UV Hubble Space Telescope observations combined with our long term radio monitoring seem to indicate that episodes of relativistic ejections suppress flux that is emitted at wavelengths shortward of the peak of the far UV spectral energy distribution, similar to what has been observed in radio loud quasars. Episodes of strong jet production also seem to suppress the high ionization broad absorption line wind seen in weak jet states. We found a statistically significant increase $(\sim 25 \%)$ of the $3-12 \mathrm{keV}$ flux during the radio flare relative to a quiescent radio state. This is explained by an ultra-fast $(\sim 0.06 \mathrm{c}) \mathrm{X}$-ray absorbing photo-ionized wind that is significantly detected only in the low radio state (similar to Galactic black holes). Mrk 231 is becoming more radio loud. We found that the putative parsec scale radio lobe doubled in brightness in 9 years. Furthermore, large flares are more frequent with 3 major flares occurring at $\sim 2$ year intervals.
\end{abstract}

Subject headings: quasars: absorption lines — galaxies: jets — quasars: general — accretion, accretion disks — black hole physics

\footnotetext{
${ }^{1}$ CSIRO Astronomy and Space Science, Kensington, WA 6151, Australia

${ }^{2} 1415$ Granvia Altamira, Palos Verdes Estates CA, USA 90274 and ICRANet, Piazza della Repubblica 10 Pescara 65100, Italy, brian.punsly1@verizon.net

${ }^{3}$ Centro de Astrobiologia (CAB) ESA - European Space Astronomy Center (ESAC)

${ }^{4}$ Department of Physics and Astronomy, University of Manitoba, Winnipeg, MB R3T 2N2 Canada

${ }^{5}$ School of Physics \& Astronomy, Rochester Institute of Technology, Rochester, NY 14623, USA

${ }^{6}$ ICRAR-Curtin University, GPO Box U1987, Perth, Western Australia, 6102, Australia
} 


\section{Introduction}

Mrk231, at a redshift of $z=0.042$, is a nearby radio quiet quasar (RQQ) with a relativistic jet (Reynolds et al. 2009). The jet is extremely powerful for a RQQ during flare states with a kinetic luminosity estimated to be $\sim 3 \times 10^{43} \mathrm{ergs} \mathrm{s}^{-1}$ for previous flares. Consequently, we have been monitoring the radio behavior at $\sim 20 \mathrm{GHz}$ for the last 7 years. Since 2011, we have detected 3 large blazar-like flares (see Reynolds et al. (2013b)) with flux densities $\geq 200 \mathrm{mJy}$ at $\sim 20 \mathrm{GHz}$. The last of these major flares initiated a target of opportunity four-epoch VLBA (Very Long Baseline Array) observation. We were also afforded director's discretionary time for three observations with the NuSTAR X-ray telescope and two observations with the XMM X-ray telescope.

Mrk 231 is a unique laboratory for studying the multiple aspects that are associated with the quasar phenomenon. It is one of a small number of RQQs that have clearly exhibited episodes of relativistic jet formation (Brunthaler et al 2000; Blundell et al 2003). It also supports broad absorption line (BAL) winds. We use the original definition of BAL quasars (BALQSOs) as quasars with UV absorbing gas that is blue shifted at least $5,000 \mathrm{~km} / \mathrm{s}$ relative to the QSO rest frame and displaying a spread in velocity of at least $2,000 \mathrm{~km} \mathrm{~s}^{-1}$, (Weymann et al. 1991). This definition excludes the so-called "mini-BALQSOs," with the BALNicity index $=0$ (Weymann 1997). This definition is preferred here since miniBALQSOs tend to resemble non-BALQSOs more than BALQSOs in many spectral and broadband properties (Punsly 2006; Zhang et al. 2010; Bruni et al. 2013; Havashi et al. 2013). A nonzero BALNicity index is extraordinary considering that the existence of large scale jets and BAL winds are almost mutually exclusive. The propensity for suppressed large scale emission increases strongly with BALnicity index (Becker et al. 2000, 2001). Thus, episodes of relativistic jet production in Mrk231 allow us to explore the interplay between BAL winds and jets. Mrk 231 is exceptional in that it tends to vacillate between radio quiet and radio loud behaviors. In particular, in this study we find states of weak jet activity have UV characteristics that differ from those of the states of high jet activity. Based on limited data, the weak jet state shows evidence of high ionization BAL winds, as noted above, a property associated with radio quiet quasars. In the states of high jet activity, the evidence of a high ionization BAL wind disappears and the spectral energy distribution steepens beyond its peak in the far UV, a property common to radio loud quasars (Telfer et al. 2002; Punsly 2015). This transition between high jet activity and low jet activity occurs on very short time scales in some Galactic black holes (Klein-Wolt et al. 2002; Prat et al. 2010). Consequently, these stellar mass objects form the basis of our physical understanding of the state change (both back and forth) between a jetted black hole accretion system to a non-jetted black hole accretion system. It is not clear that the trends observed in Galactic black holes extrapolate many orders of magnitude in both time and space to quasars. This was the mo- 
tivation for our previous high frequency $(15 \mathrm{GHz}, 22 \mathrm{GHz}$ and $43 \mathrm{GHz}$ ) VLBA observations (Reynolds et al. 2009). Our previous VLBA observation detected a strong $22 \mathrm{GHz}$ flare that emerged from the core between epochs 2006.07 and 2006.32 ( $>150 \%$ increase in less than 3 months). However, we learned that the putative ejecta faded very rapidly and required both high resolution ( $43 \mathrm{GHz}$ ) and high sensitivity. Thus, we recognised the need to observe a strong flare that was still growing and to improve the sensitivity by choosing a higher data sampling rate, 2 Gbps (as opposed to the $256 \mathrm{Mbps}$ that was previously available). This observation challenged the limits of the VLBA and our efforts were designed to maximize the likelihood of successful phase-referencing and the subsequent self-calibration of the weak target source. In order to determine the initiation of a Target of Opportunity (ToO) for our VLBA observations, we began monitoring Mrk 231 at $22 \mathrm{GHz}$ in 2009, switching to 17.6 GHz in 2012. The details of our monitoring program and the discovery of a large flare were previously reported (Reynolds et al. 2013b).

The paper is organized as follows. Section 2 describes the observational details of our monitoring. In Section 3, we describe the VLBA observations that were triggered on 2015 March 31. There were four observations at 8.4, 15.2, 22.2 and $43 \mathrm{GHz}$ that were spread out over 15 weeks. In Section 4, we describe Hubble Space Telescope (HST) observations of the far UV spectrum of Mrk 231 that serendipitously align with three epochs of our radio monitoring. In Section 5, we discuss the X-ray telescope observations that were granted after our VLBA ToO, three NuSTAR observations and two XMM observations. Throughout this paper, we adopt the following cosmological parameters: $H_{0}=71 \mathrm{~km} \mathrm{~s}^{-1} \mathrm{Mpc}^{-1}, \Omega_{\Lambda}=0.73$ and $\Omega_{m}=0.27$.

\section{The Radio Monitoring}

Reynolds et al. (2013b) reported the methods of our radio monitoring program. In summary, the program utilized long term monitoring with the VLA (Very Large Array) and EVLA (Expanded Very Large Array) at $22 \mathrm{GHz}$ from 2009 to 2012 and from 2013 to 2015 AMI (Arcminute Microkelvin Imager; Zwart et al. 2008) at $13.5-18 \mathrm{GHz} 1$. The long term monitoring data from Reynolds et al. (2013b) are plotted in the top frame of Figure 1. This light curve incorporates historical data both from the literature (McCutcheon and Gregory

\footnotetext{
${ }^{1}$ The Arcminute Microkelvin Imager consists of two radio interferometric arrays located in the Mullard Radio Astronomical Observatory, Cambridge, UK (Zwart et al. 2008). Observations occur between 13.9 and 18.2 GHz in six frequency channels. The Small Array (AMI-SA) consists of ten $3.6 \mathrm{~m}$ diameter dishes with a maximum baseline of $20 \mathrm{~m}$, with an angular resolution of $3^{\prime}$, while the Large Array (AMI-LA) comprises eight $12.6 \mathrm{~m}$ diameter dishes with a maximum baseline of $110 \mathrm{~m}$, giving an angular resolution of 0.5 .
} 

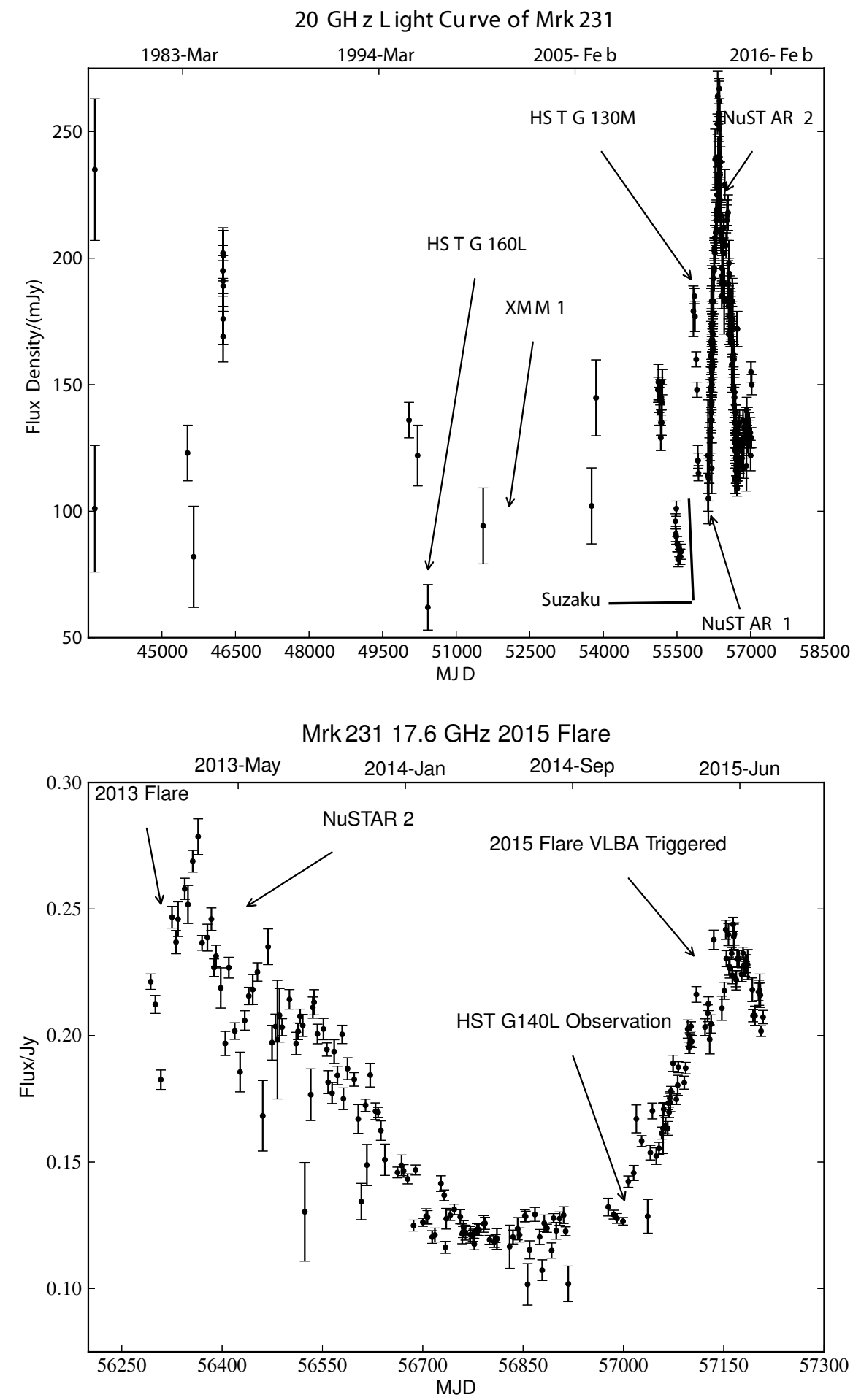

Fig. 1. - The top frame is the historic $\sim 20 \mathrm{GHz}$ light curve from Revnolds et al. (2013b) with the timing of observations in other energy bands that are discussed in this paper superimposed. The bottom frame is an AMI 17.6 GHz light curve showing the initiation of the radio flare discussed in this article. 
1978; Edelson 1987; Ulvestad et al. 1999a, b; Reynolds et al. 2009) and from the VLA public archive (project codes AB783, AN030, AU015). We have superimposed the times of relevant observations that we will reference in the following. The notable features of the historical light curve are the major flare of 2013 that was monitored with AMI and analyzed previously (Reynolds et al. 2013b). It was shown that this is similar to blazar flares (rise time, decay and relative magnitude), but on a smaller scale. The unexpected steady rise of flux failed to meet our trigger criteria (which were then revised) and we missed this opportunity to study a flare in progress. The other important feature is the HST Faint Object Spectrograph (FOS) observation with the G160L grating on 1996 November 21, just 14 days before the historically low VLA flux density measurement (Ulvestad et al. 1999a). This fortuitous circumstance will form an important part of our analysis in Section 4. Another fortuitous circumstance is the HST Cosmic Origins Spectrograph (COS) observation with the G130M grating on 2011 October 15 reported in Veilleux. Trippe and Hamann et al. (2013) that occurred during a $22 \mathrm{GHz}$ flare.

The bottom frame of Figure 1 is the $17.6 \mathrm{GHz}$ (the highest useable frequency) light curve from our more recent AMI monitoring. The 2013 flare is on the left hand side. The outlier data points with large error bars during the flare decay are affected by poor weather (precipitation). We were unable to correct for the weather and they should not be considered as evidence of rapid flux changes. The 2015 flare appears on the right hand side of the figure. The VLBA observations (and subsequent coordinated X-ray observations) were triggered on 2015 March 31. The flare was still rising and approaching the peak flux density, so the timing was ideal for our program - the maximum brightness for study during the flare rise. Knowing that the detected fluxes were going to be small at the crucial frequency of $43 \mathrm{GHz}$, achieving maximum brightness was essential to the success of the observation. The other interesting feature of the light curve is timing of the HST COS observation with the G140L grating on 2014 December 13. The flare was already beginning at this time at $17.6 \mathrm{GHz}$, and as discussed in the next two sections, due to synchrotron self absorption (SSA) optical depth affects the $43 \mathrm{GHz}$ flare began at least a few days to weeks earlier. Again, this is fortuitous timing of the HST observation. All three of the far UV HST observations serendipitously occurred during times of radio monitoring.

\section{VLBA Observations}

The VLBA project, BR205, successfully acquired data from all ten stations during each of the four epochs of observation. The data were correlated on the VLBA correlator (Deller et al. 2011) and calibrated with NRAO's Astronomical Imaging Processing System 


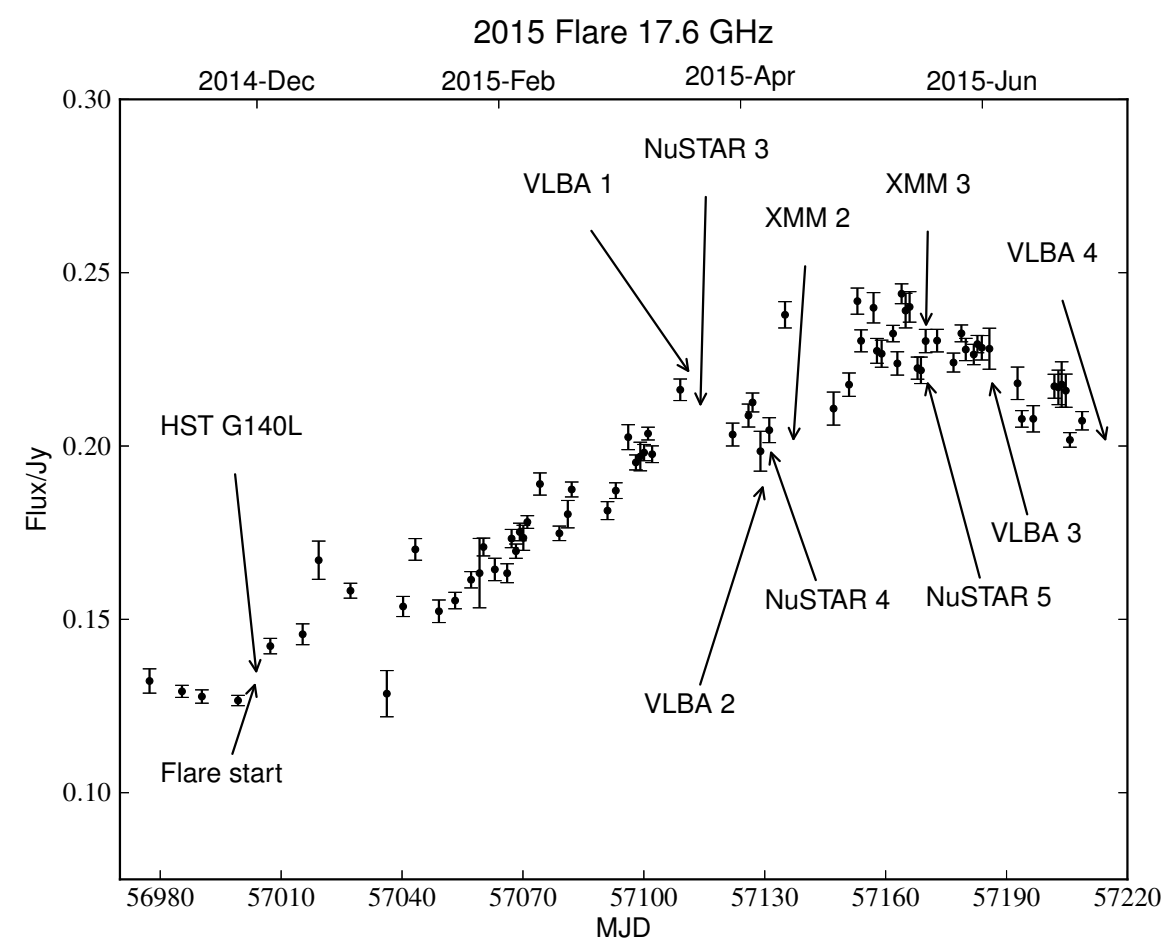

Fig. 2.- The AMI 17.6 GHz light curve of the 2015 flare. Note the dates of the observations discussed in this article. 
using the ParselTongue interface (Kettenis et al. 2006).

Our observations were phase-referenced to J1302+5748 (1.3 from Mrk 231) at 8.4, 15, 22, and $43 \mathrm{GHz}$, following the strategies described in Reynolds et al. (2009) for project BP124. J1311+5513 ( $~ 80$ mJy of unresolved flux density at $43 \mathrm{GHz}$ ) was used as a secondary calibrator to check the quality of the phase referencing. For each epoch of our VLBA monitoring, two 4-hour observations with almost continuous recording at $2 \mathrm{Gbps}(256 \mathrm{MHz}$ bandwidth, dual polarizations) were acquired in order to achieve the SNR (Signal to Noise Ratio) required to resolve the putative sub-components of the $43 \mathrm{GHz}$ core and to maximize the likelihood of successful phase-referencing and the subsequent self-calibration of the weak target source, Mrk 231. For scheduling purposes, the 4 hour blocks were split into two consecutive days except for the last epoch in which both blocks occurred in a continuous time interval. After calibration overhead, this resulted in 2.6 hours on Mrk 231 per epoch, that was split as $0.25,0.35,0.8$, and 1.2 hours at frequencies of $8,15,22$, and $43 \mathrm{GHz}$, respectively. All 4 stokes parameters were correlated and we were able to achieve naturally weighted image RMS values in Stokes I of approximately 50, 100, 50, and $70 \mu \mathrm{Jy}$ at 8.4, 15, 22 , and $43 \mathrm{GHz}$ respectively.

The observations were generally successful except for one major unexpected technical issue. Due to scheduling constraints, we could not be granted a continuous 8 hour observation. The compromise of splitting the observation into 4 hour increments on consecutive days proved deleterious in epochs 2 and 3 as the two segments were observed at almost the same LST (Local Sidereal Time). The resulting beam was poorly shaped due to insufficient $u-v$ coverage and the resolution was inadequate for resolving structures along the jet. We analyze the resulting data even though this circumstance made it impossible to directly monitor motion along the jet (a primary objective of the program).

Figure 2 is the $17.6 \mathrm{GHz}$ light curve of the 2015 flare. The flare initiates at the beginning to the middle of December 2014. The relevant VLBA, HST, XMM and NuSTAR observations times are indicated relative to the light curve. Table 1 lists the results of our model fits to the VLBA observation. There are four components listed in column (1), the core, K1: a stationary secondary $0.8 \mathrm{pc}$ from the core that has been detected in previous VLBA observations and two new components, K2 and K3. We discuss whether K2 and K3 might be the same component in this section. New components are only detected in epochs 1 and 4, presumably due to an inopportune beam shape in epochs 2 and 3. Column (2) is the date of the observation. The flux density of the component is listed in column (3). The uncertainty in the flux density is listed in the column (4). The next four columns are the $\mathrm{X}$ (east-west) and Y (north-south) coordinates (relative to the phase centre) of each fitted component and the associated uncertainty. Notice that the origin (phase centre) is close to K1, not the 
Table 1: Summary of Model Fits to VLBA Observations

\begin{tabular}{|c|c|c|c|c|c|c|c|c|c|c|c|}
\hline 1 & 2 & 3 & 4 & 5 & 6 & 7 & 8 & 9 & 10 & 11 & 12 \\
\hline \multirow[t]{3}{*}{ Component } & epoch & Flux & Flux & $\mathrm{X}$ & $\mathrm{X}$ & Y & $\mathrm{Y}$ & Major & Axial & $\mathrm{PA}$ & Frequency \\
\hline & & $\begin{array}{c}\text { Density } \\
(\mathrm{Jy})\end{array}$ & $\begin{array}{c}\text { Density } \sigma \\
(\mathrm{Jy})\end{array}$ & (mas) & $\begin{array}{c}\sigma \\
(\mathrm{mas})\end{array}$ & (mas) & $\begin{array}{c}\sigma \\
(\mathrm{mas}) \\
\end{array}$ & $\begin{array}{l}\text { Axis } \\
\text { (mas) }\end{array}$ & Ratio & $(\mathrm{deg})$ & $(\mathrm{GHz}))$ \\
\hline & & & & & Epoch & 1 & & & & & \\
\hline K1 & 2015.247 & 0.215 & 0.011 & 0.171 & 0.001 & 0.101 & 0.001 & 0.33 & 0.83 & -80.1 & 8.42 \\
\hline Core & 2015.247 & 0.071 & 0.004 & 1.205 & 0.002 & 0.582 & 0.003 & 0.17 & 0.93 & 48.4 & 8.42 \\
\hline $\mathrm{K} 1$ & 2015.247 & 0.093 & 0.005 & 0.186 & 0.001 & 0.103 & 0.002 & 0.29 & 0.77 & -85.5 & 15.3 \\
\hline Core & 2015.247 & 0.094 & 0.005 & 1.259 & 0.001 & 0.601 & 0.002 & 0.12 & 0.00 & 85.8 & 15.3 \\
\hline $\mathrm{K} 1$ & 2015.247 & 0.052 & 0.004 & 0.148 & 0.002 & 0.149 & 0.003 & 0.28 & 0.72 & -85.3 & 22.2 \\
\hline Core & 2015.247 & 0.083 & 0.006 & 1.236 & 0.001 & 0.657 & 0.002 & 0.11 & 0.63 & 88.2 & 22.2 \\
\hline Core & 2015.247 & 0.045 & 0.005 & 1.253 & 0.001 & 0.613 & 0.001 & 0.10 & 0.00 & 49.6 & 43.1 \\
\hline K1 & 2015.247 & 0.018 & 0.002 & 0.137 & 0.002 & 0.097 & 0.004 & 0.29 & 0.52 & 73.5 & 43.1 \\
\hline \multirow[t]{2}{*}{$\mathrm{K} 2$} & 2015.247 & 0.002 & 0.001 & 0.939 & 0.017 & 0.449 & 0.030 & 0.00 & 1.00 & 0.00 & 43.1 \\
\hline & & & & & Epoch & $\overline{2}$ & & & & & \\
\hline$\overline{\mathrm{K} 1}$ & 2015.296 & 0.228 & 0.011 & 0.285 & 0.001 & 0.095 & 0.001 & $\overline{0.45}$ & 0.62 & $\overline{768.3}$ & 8.42 \\
\hline Core & 2015.296 & 0.069 & 0.003 & 1.344 & 0.004 & 0.587 & 0.004 & 0.18 & 0.00 & -58.2 & 8.42 \\
\hline $\mathrm{K} 1$ & 2015.296 & 0.098 & 0.005 & 0.186 & 0.002 & -0.023 & 0.002 & 0.26 & 0.88 & -83.2 & 15.3 \\
\hline Core & 2015.296 & 0.094 & 0.005 & 1.250 & 0.005 & 0.471 & 0.002 & 0.07 & 0.61 & -14.9 & 15.3 \\
\hline K1 & 2015.296 & 0.057 & 0.004 & 0.225 & 0.002 & 0.080 & 0.002 & 0.27 & 0.95 & 52.9 & 22.2 \\
\hline Core & 2015.296 & 0.083 & 0.006 & 1.295 & 0.001 & 0.580 & 0.002 & 0.17 & 0.45 & 24.2 & 22.2 \\
\hline Core & 2015.296 & 0.044 & 0.004 & 1.315 & 0.002 & 0.580 & 0.002 & 0.14 & 0.00 & 67.5 & 43.1 \\
\hline \multirow[t]{2}{*}{$\mathrm{K} 1$} & 2015.296 & 0.018 & 0.002 & 0.214 & 0.005 & 0.073 & 0.004 & 0.25 & 0.57 & 88.1 & 43.1 \\
\hline & & & & & Epoch & $\overline{3}$ & & & & & \\
\hline$\overline{\mathrm{K} 1}$ & 2015.452 & 0.259 & 0.013 & 0.186 & 0.001 & 0.007 & 0.001 & 0.43 & 0.62 & 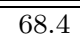 & 8.42 \\
\hline Core & 2015.452 & 0.070 & 0.004 & 1.237 & 0.015 & 0.485 & 0.005 & 0.33 & 0.00 & 19.9 & 8.42 \\
\hline K1 & 2015.452 & 0.104 & 0.005 & 0.137 & 0.003 & -0.052 & 0.002 & 0.33 & 0.57 & 26.9 & 15.3 \\
\hline Core & 2015.452 & 0.077 & 0.004 & 1.183 & 0.004 & 0.422 & 0.003 & 0.31 & 0.00 & 45.4 & 15.3 \\
\hline K1 & 2015.452 & 0.057 & 0.004 & 0.110 & 0.002 & -0.083 & 0.002 & 0.24 & 0.87 & 86.4 & 22.2 \\
\hline Core & 2015.452 & 0.055 & 0.004 & 1.175 & 0.002 & 0.401 & 0.002 & 0.14 & 0.32 & 21.3 & 22.2 \\
\hline Core & 2015.452 & 0.027 & 0.003 & 1.233 & 0.003 & 0.408 & 0.003 & 0.08 & 0.38 & 78.7 & 43.1 \\
\hline \multirow[t]{2}{*}{$\mathrm{K} 1$} & 2015.452 & 0.020 & 0.002 & 0.150 & 0.004 & -0.092 & 0.004 & 0.24 & 0.87 & 86.4 & 43.1 \\
\hline & & & & & Epoch & 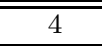 & & & & & \\
\hline$\overline{\mathrm{K} 1}$ & 2015.529 & 0.262 & 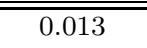 & 0.155 & 0.002 & 0.006 & 0.004 & $\overline{0.29}$ & 0.71 & -64.3 & 8.42 \\
\hline Core & 2015.529 & 0.063 & 0.003 & 1.181 & 0.010 & 0.482 & 0.015 & 0.21 & 0.00 & -8.2 & 8.42 \\
\hline K1 & 2015.529 & 0.096 & 0.005 & 0.085 & 0.002 & -0.058 & 0.003 & 0.28 & 0.44 & -88.1 & 15.3 \\
\hline Core & 2015.529 & 0.048 & 0.002 & 1.146 & 0.004 & 0.426 & 0.007 & 0.18 & 0.00 & 63.0 & 15.3 \\
\hline K1 & 2015.520 & 0.066 & 0.005 & 0.066 & 0.001 & -0.071 & 0.002 & 0.24 & 0.54 & -80.3 & 22.2 \\
\hline Core & 2015.529 & 0.042 & 0.003 & 1.142 & 0.002 & 0.423 & 0.003 & 0.08 & 0.00 & 64.1 & 22.2 \\
\hline Core & 2015.529 & 0.032 & 0.003 & 1.205 & 0.002 & 0.422 & 0.004 & 0.21 & 0.00 & 67.0 & 43.1 \\
\hline K1 & 2015.529 & 0.025 & 0.002 & 0.071 & 0.003 & -0.097 & 0.005 & 0.22 & 0.69 & -69.5 & 43.1 \\
\hline K3 & 2015.529 & 0.003 & 0.001 & 1.049 & 0.027 & 0.247 & 0.042 & 0.00 & 1.00 & 0.00 & 43.1 \\
\hline
\end{tabular}


core, due to the fact that the early position determination was done at low frequency where the steep spectrum K1 component dominates (Reynolds et al. 2009). Columns (9) - (11) describe the fitted elliptical Gaussian or point source models for each component. We list the major axis, the axial ratio and the position angle. The last column is the frequency of observation.

In Figure 3, we show the radio images for 8.4, 15.3 and 22.2 GHz. These images are relatively low resolution for this study and do not indicate any interesting nuclear morphology. The resultant flux densities of the core are important for determining the core spectrum in each epoch. These images are similar to the low resolution images in Reynolds et al. (2009). At $8.4 \mathrm{GHz}$, component $\mathrm{K} 1$ is dominant and the source is only partially resolved. At $15.3 \mathrm{GHz}$, the two components are resolved with roughly equal flux densities. Finally, at $22.2 \mathrm{GHz}$, the core is brighter except during the flare decay. The differences in apparent morphology at a given frequency are primarily a result of beam shape. Notice the elongated beam shape along the direction from the core to K1 in epochs 2 and 3. This greatly de-

graded the resolution along the jet direction in the critical $43.1 \mathrm{GHz}$ observations during these epochs.

\subsection{Nuclear Structure}

Figure 4 shows the $43.1 \mathrm{GHz}$ VLBA images from the four epochs. The components $\mathrm{K} 2$ and $\mathrm{K} 3$ are the new ejections that are partially resolved in epochs 1 and 4. Detecting these putative ejections was a major goal of these observations. As noted earlier, the higher sensitivity observations afforded by the new VLBA backend systems improved the ability to phase reference and detect these ejecta compared to our 2006 observations. The new component could not be detected in epochs 2 and 3 due to an unfortunate beam shape elongated along the jet direction: the direction that needs the highest resolution in order to resolve new components ejected from the core. The ejected component flux density in the two epochs is only 2-3 mJy. We identify the partially resolved component in epochs 1 and 4 as $\mathrm{K} 2$ and $\mathrm{K} 3$, respectively.

Even though a large amount of information was lost due to poor resolution along the jet direction in epochs 2 and 3, we did anticipate the additional information that can be found in the core spectrum with a multi-frequency observation plan. This aspect was achieved as all 10 VLBA stations successfully observed in all epochs. The spectra of the unresolved core in each epoch are shown in Figure 5. In Reynolds et al. (2009), we used the detailed nature of the spectral shape to argue that the spectrum of the radio core was most likely a power law synchrotron spectrum that was seen through a SSA screen as opposed to a synchrotron 

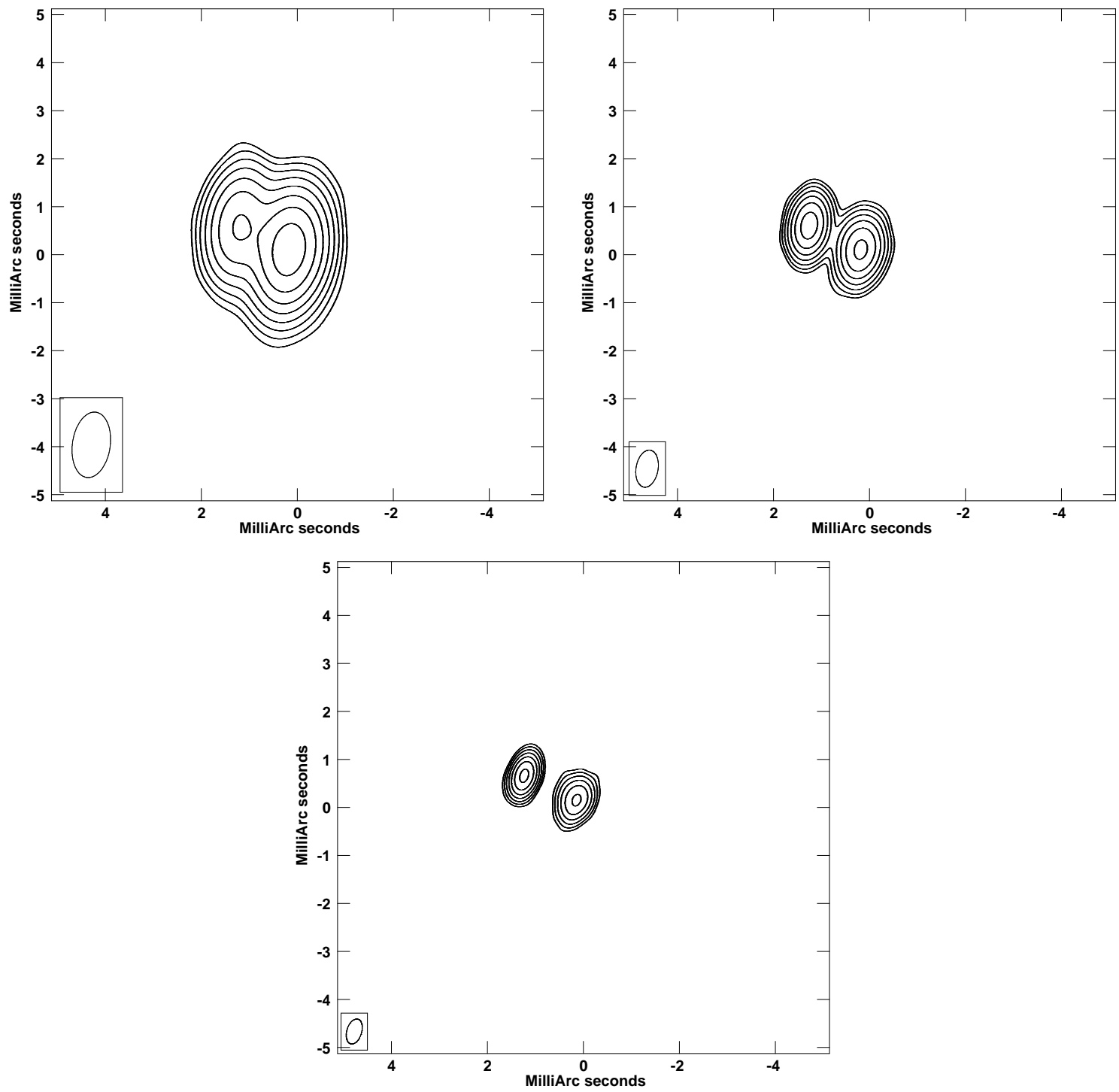

Fig. 3.- VLBA images at 8.4 (top left), 15.3 (top right) and $22.2 \mathrm{GHz}$ (bottom) from epoch 1. In each case the bottom contour is $0.1 \mathrm{mJy} /$ beam increasing in factors of 2 . The data have been weighted with Briggs's weighting scheme (Briggs 1995) using a robustness parameter of 0 . 

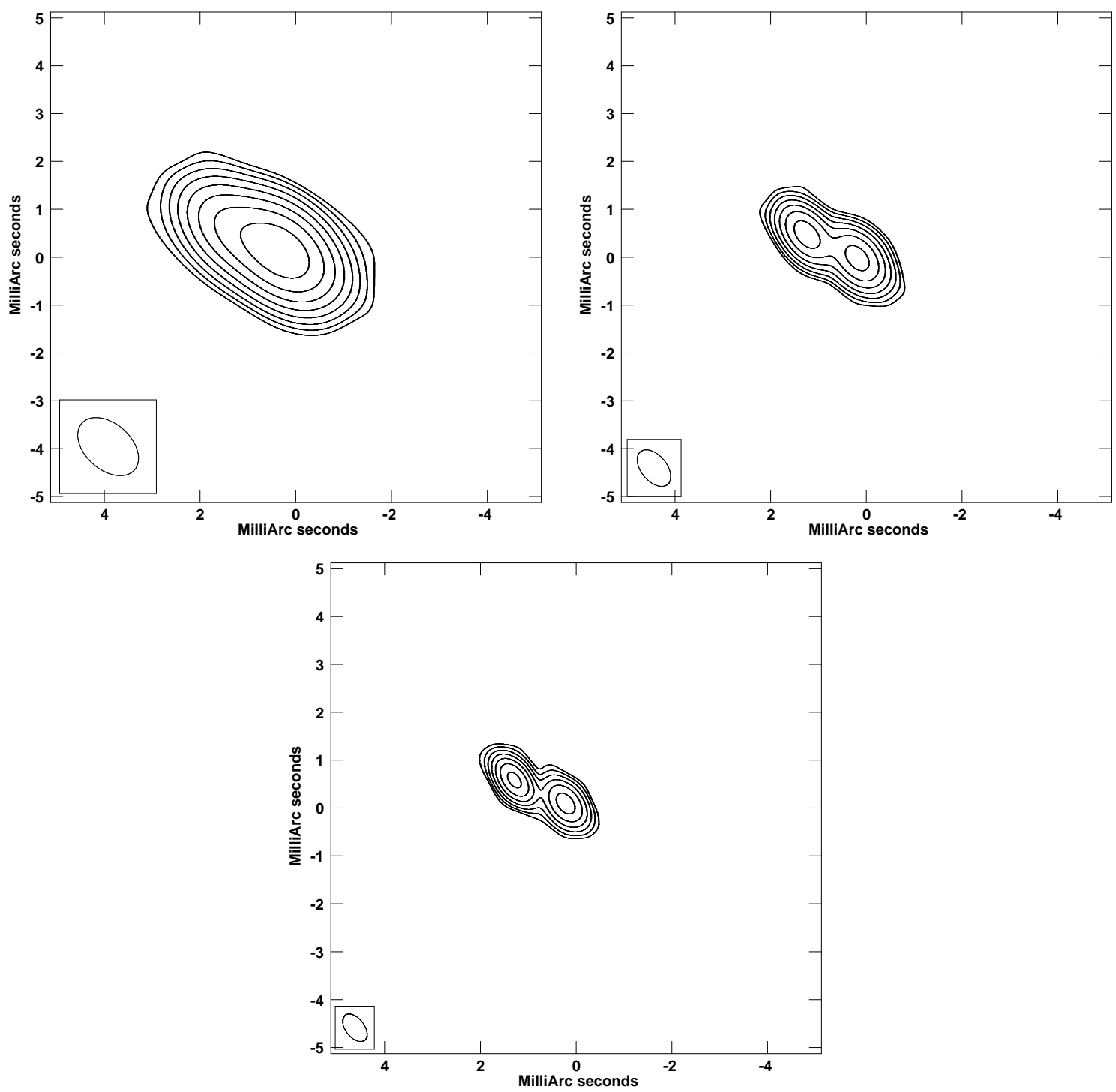

Fig. 3.- (continued) VLBA images at 8.4 (top left), 15.3 (top right) and $22.2 \mathrm{GHz}$ (bottom) from epoch 2. In each case the bottom contour is $0.1 \mathrm{mJy} /$ beam increasing in factors of 2. The data have been weighted with Briggs's weighting scheme (Briggs 1995) using a robustness parameter of 0 . 

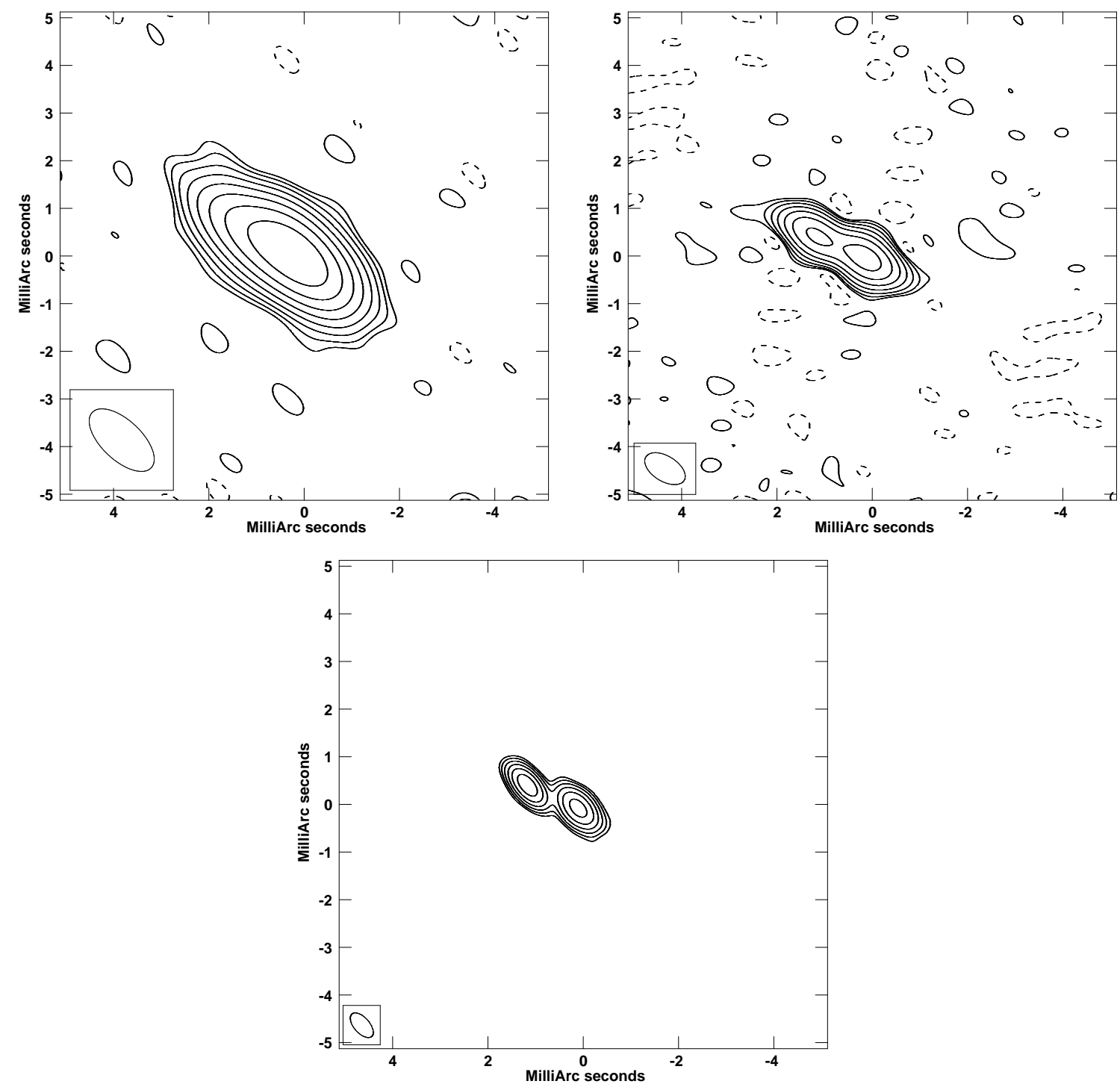

Fig. 3.- (continued) VLBA images at 8.4 (top left), 15.3 (top right) and $22.2 \mathrm{GHz}$ (bottom) from epoch 3. In each case the bottom contour is $0.1 \mathrm{mJy} /$ beam increasing in factors of 2. The data have been weighted with Briggs's weighting scheme (Briggs 1995) using a robustness parameter of 0 . 

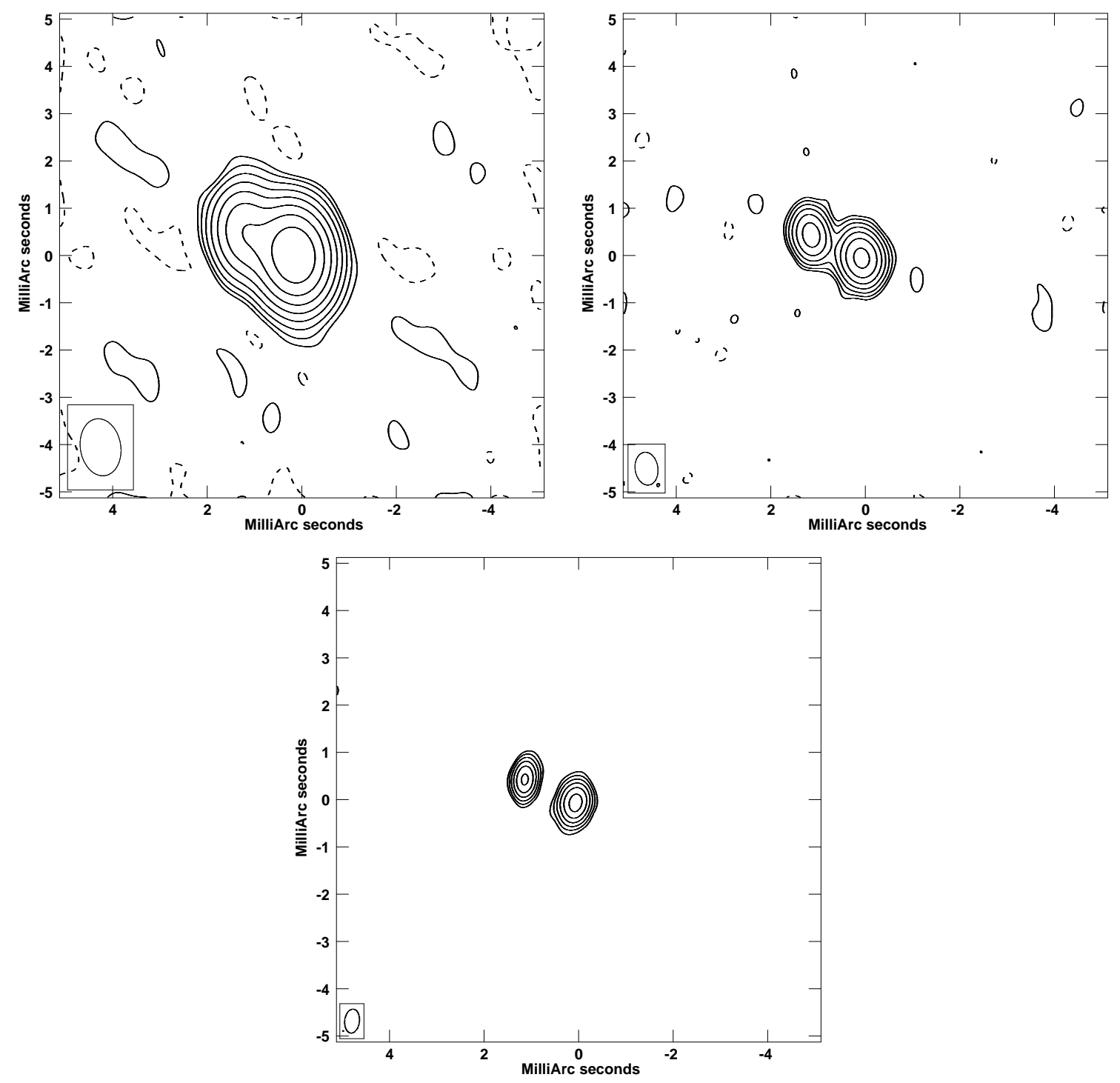

Fig. 3.- (continued) VLBA images at 8.4 (top left), 15.3 (top right) and $22.2 \mathrm{GHz}$ (bottom) from epoch 4. In each case the bottom contour is $0.1 \mathrm{mJy} /$ beam increasing in factors of 2. The data have been weighted with Briggs's weighting scheme (Briggs 1995) using a robustness parameter of 0 . 

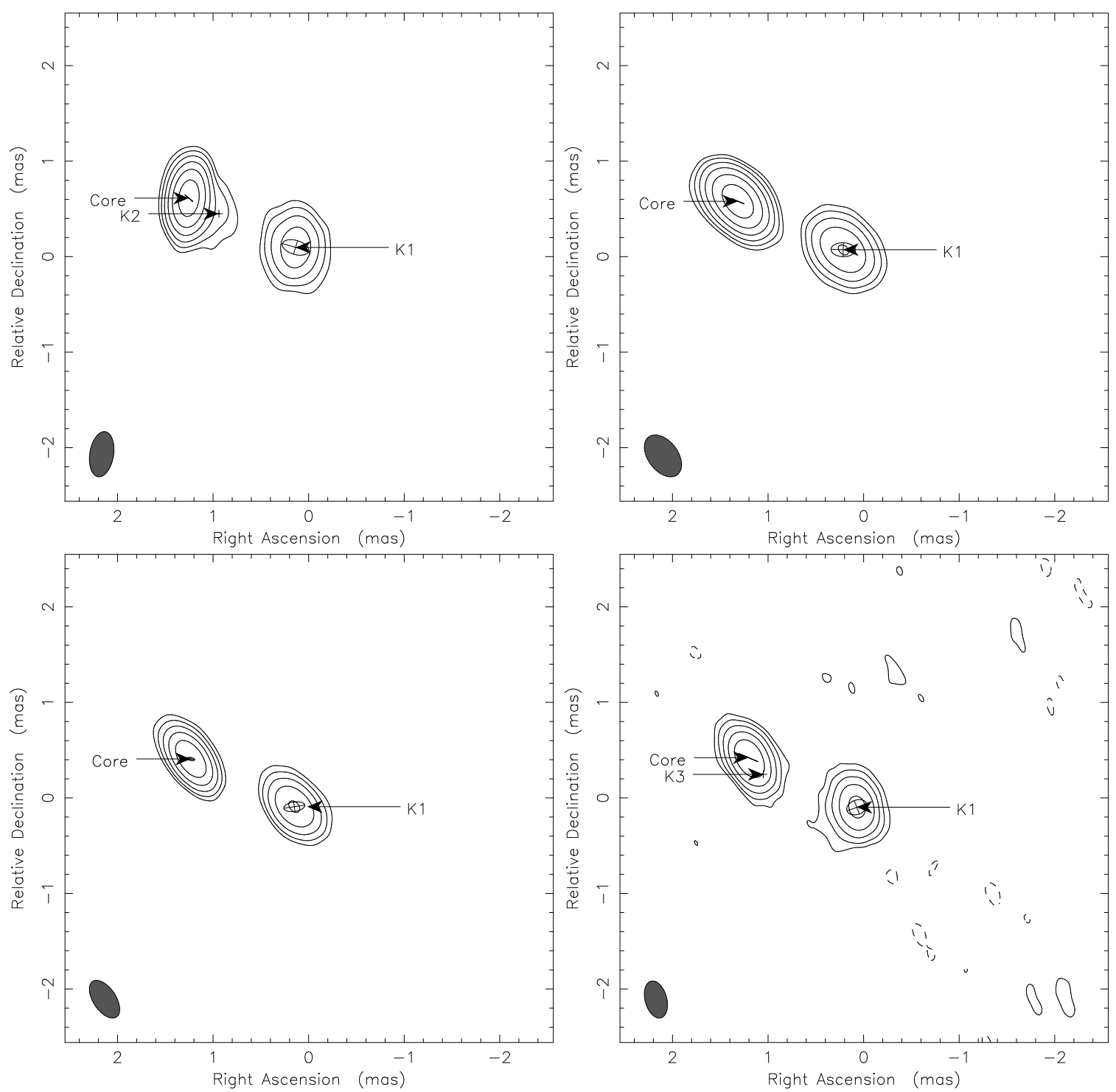

Fig. 4.- Naturally weighted VLBA images at $43.1 \mathrm{GHz}$. The top row from left to right are epochs 1 and 2. The bottom row from left to right are epochs 3 and 4. The bottom contour is at $0.085 \mathrm{mJy} /$ beam in each case and contours increase in factors of 2 . The crosses and ellipses with inscribed crosses denote the locations of point source components and the locations and dimensions of the Gaussian elliptical components, respectively. Note that the major axis of the elliptical Gaussian model for the core tends to point along the jet direction. It is also very elongated in this direction during the earlier stages of the flare. 


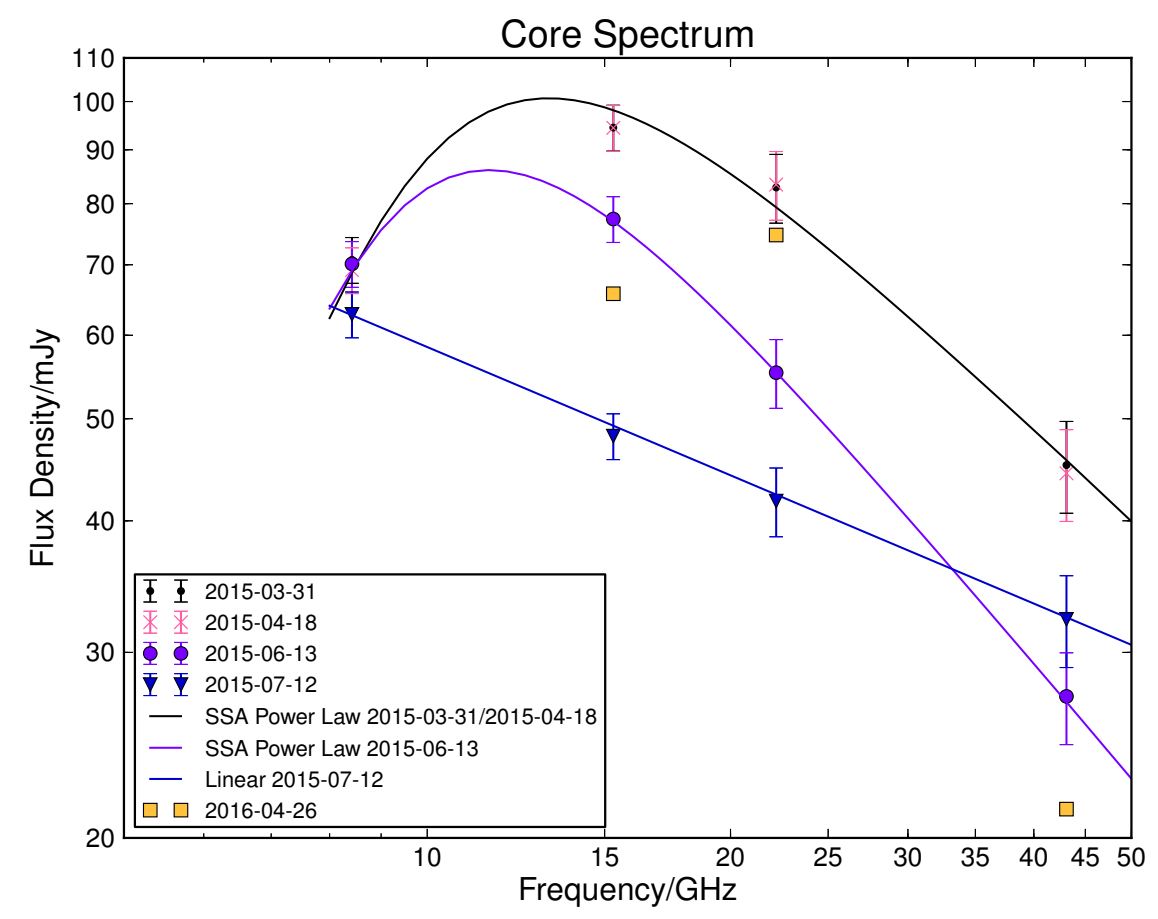

Fig. 5. - The spectrum of the unresolved radio core in the four epochs. The data are from Table 1. The continuous curves are SSA power law fits to the data. We have also added the flux density of the 2006.32 unresolved nucleus for historical reference to the flare described in (Reynolds et al. 2009). 


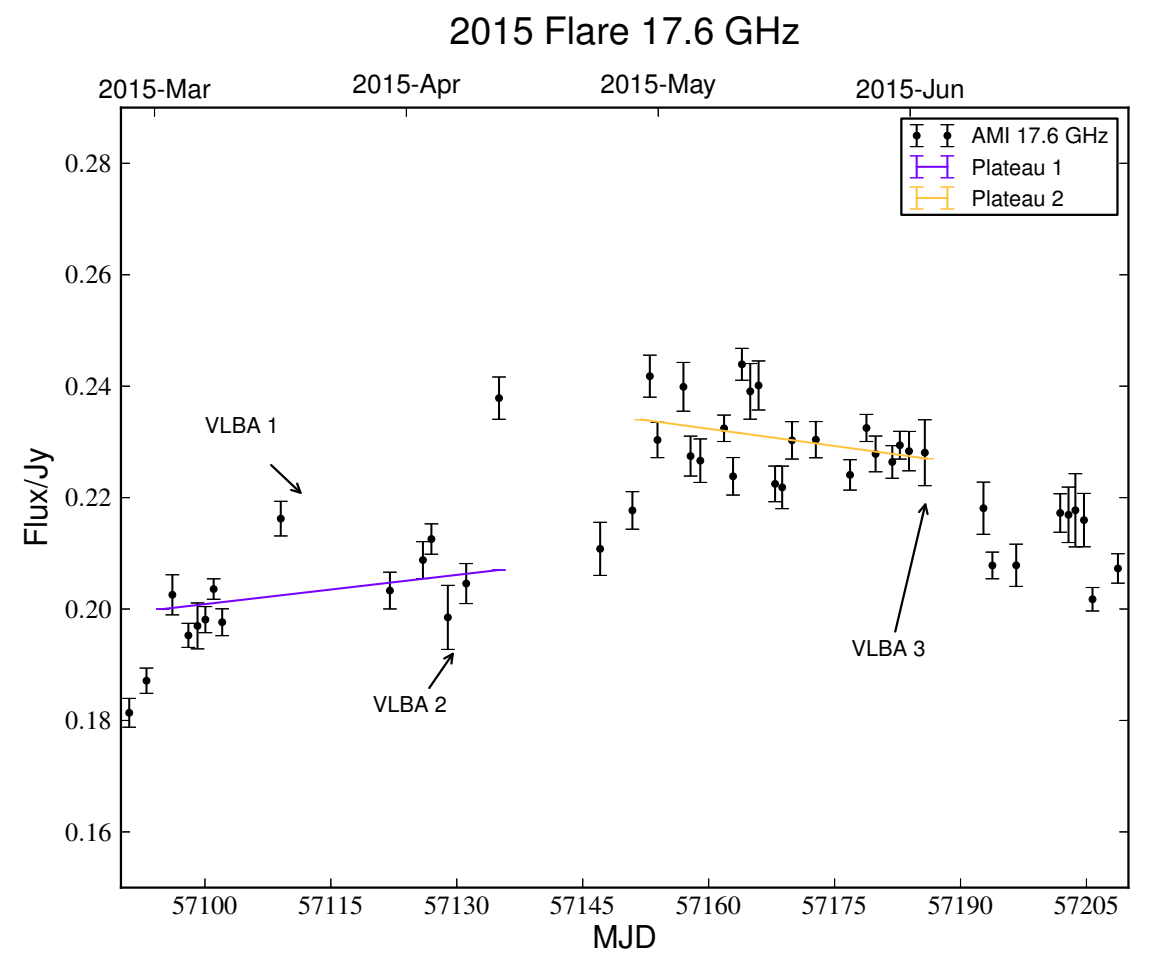

Fig. 6.- A close up of the active plateau regions of the light curve. These are high states associated with the peak of jet activity. 
power law spectrum that was seen through an opaque screen caused by free-free absorption. The spectrum in epoch 1 is consistent with this interpretation.

The plots in Figure 5 show synchrotron self-absorbed (SSA) power law fits to the first three epochs - a simple spectral model has been used to describe previous observations (Reynolds et al. 2009). The first two epochs have surprisingly similar spectra and fits. The spectral index is $\alpha=0.91$ and the SSA optical depth is unity at $11.2 \mathrm{GHz}$ (where $\alpha$ is defined in terms of flux density as $F_{\nu} \sim \nu^{-\alpha}$ ). The third epoch has $\alpha=1.13$ and an optical depth of unity at $10.4 \mathrm{GHz}$. The trend is ostensibly that which would be expected as a source expands (adiabatic cooling) and/or is synchrotron cooled or is Compton cooled by accretion disk photons. In this scenario, the peak emission should shift to lower and lower frequency and the spectrum should steepen at high frequency (the highest energy electrons cool first) (Moffet 1975; Tucker 1975; Ginzburg and Syrovatskii 1969). Oddly, epoch four is a pure power law with no turnover due to SSA absorption and $\alpha=0.41$. There is a fundamental physical change in the nucleus between epoch 3 and 4 . The most curious aspect of epoch 4 , considering the fading of the flare, is that the spectrum is relatively flat. It seems to represent a disjoint epoch of low level power law emission that supersedes the cooling plasmoid phase that occurs between epochs 2 and 3. We have included the nuclear flux densities from the 2006.32 flare that were given in Reynolds et al. (2009) for comparison purposes. In the context of a simple SSA power law model, the nucleus was more self-absorbed in 2006 with a steeper power law spectral index.

Perhaps the most unexpected finding is that the nuclear spectrum is unchanged within the measurement accuracy of the VLBA between epochs 1 and 2. In 18 days there is no change in the spectrum, yet the light curve shows $20 \mathrm{mJy}$ peak to peak variation at 17.6 GHz in the intervening time frame. Presumably, considering the size of the error bars on the AMI data it is quite possible that the intrinsic flux was actually quite stable during this time frame and possibly even back to the middle of March 2015, with only a few mJy variation (approximately $205 \mathrm{mJy}-210 \mathrm{mJy}$ ) and the triggering observation on $2016 \mathrm{March}$ $27(216 \mathrm{mJy})$ was a statistically extreme variation arising from systematic errors. This is the only consistent explanation of the combined AMI and VLBA data from mid-March to mid April. The implication is there was a broad one month plateau in the light curve that corresponded to a sustained epoch of jet activity. With this interpretation, it is very evident from the AMI light curve in Figure 2 that a new epoch of jet behavior commences immediately after the epoch 2 VLBA observation, more variable with larger flux densities, $225 \mathrm{mJy}-240 \mathrm{mJy}$. The interpretation of the peak of jet activity during the 2015 flare as two distinct plateaus is illustrated in Figure 6. VLBA epochs 1 and 2 are in the middle and end of the plateau 1, respectively. This could explain the similar core spectra in Figure 5. There is no observation during plateau 2. The VLBA observation in epoch 3 appears to be 
during the very beginning of the decay of the more luminous plateau 2. Unfortunately, there is no AMI data that was available near the time of the epoch 4 observation or afterward.

\subsection{Ejected Components}

An important diagnostic of the jet activity near the source is provided by component motion. We can improve on the inherent astrometric accuracy provided by our phasereferenced observations by using the position of component $\mathrm{K} 1$ as a reference point. This is a very stationary feature. In the two epochs observed in Reynolds et al. (2009), 2006.07 and 2006.32, the distance from the core to $\mathrm{K} 1$ at $43 \mathrm{GHz}$ was 1.222 mas and 1.216 mas. In the four epochs observed in this program, the separations at $43.1 \mathrm{GHz}$, were 1.230 mas, 1.212 mas, 1.192 mas and 1.247 mas in temporal order, with an average of 1.220 mas. This equates to $<0.05$ mas of motion in 9 years. Thus, with excellent precision we consider K1 to be a fixed origin for our astrometric coordinates. We will explain the fluctuations in this separation during our four observations in the following discussion. They are likely related to beam shape and nuclear activity.

In contrast, the core is not a reliable origin for determining component motion. This is not due to SSA opacity affects as are traditionally considered in homogeneous jet models (Blandford and Köingl 1979). The spectrum is indicative of optically thin plasma at 43.1 GHz in epochs $1-3$. It is likely that resolution as a consequence of beam shape and size will determine how much of the optically thin emission is represented in the $43.1 \mathrm{GHz}$ core. In this context, it is important to recognize that there are likely multiple discrete ejections that are unresolved by the VLBA at $43.1 \mathrm{GHz}$. We can infer this from two pieces of evidence. Firstly there are the abrupt jumps in $17.6 \mathrm{GHz}$ flux density that occur in the AMI light curve in Figure 2. Secondly, the Gaussian elliptical fits to the $43.1 \mathrm{GHz}$ core in Table 1 are characterized by a major axis that is very elongated with a major axis more than half a beam width. The major axis is approximately parallel to the direction to the stationary component, K1. Considering the detection of two ejected components K2 and K3 and the abrupt jumps in the light curve, the existence of multiple components along the jet direction are a natural explanation of the $43.1 \mathrm{GHz}$ Gaussian fits to the unresolved core that are highly elongated along the jet direction. This interpretation of the nuclear fits is consistent with the core position shift that is evident in Figure 7 from epoch to epoch. Depending on the distribution and brightness of these ejecta, the centroid of the unresolved beam will move. Consider the motion of the $43.1 \mathrm{GHz}$ radio core in Figure 7 . In this plot, all of the coordinates are based on making the unresolved, stationary component K1, the true origin for astrometry. Notice that the core moves along the jet towards K1 at epoch 2. The reason 
is likely two fold. First there are possible new unresolved ejecta that might be manifested as modulations of the light curve in Figures 2 and 6. Secondly, the elongation of the beam shape along the jet direction inhibits the resolution of new and older weak components that have been ejected (i.e., component K2 from epoch 1 cannot be resolved by this beam). This inability to resolve ejecta that are directed toward $\mathrm{K} 1$ will result in a shift of the centroid of the core emission toward K1. The same effect occurs in epoch 3 as the poor resolution along the jet direction is unable to resolve the relatively strong new ejection, K3. This is likely the cause of the shift of the core position to its closest point toward K1. As the core becomes less active during the fade of the flare after epoch 3, the core drifts away from K1 as the ejecta are fewer and weaker and therefore contribute less to the unresolved base of the jet. The motion between epochs 3 and 4 is exaggerated by the elongated beam along the jet direction in epoch 3. The smaller beam along the jet direction in epoch 4 resolves out another component which we call K3. Thus, we consider the core position in epoch 4 our best estimate of the active galactic nucleus (AGN) because it is based on high resolution along the jet direction and the observation is during a state of decline of the $17.6 \mathrm{GHz}$ radio flare and there is less likely to be unresolved, strong, new components.

In the absence of sufficient resolution to track component motion in epochs 2 and 3, we use the location of the core in the relatively quiescent epoch 4 (the AGN location) in order to constrain the apparent velocity of the components. We note that the shift of the core in epoch 4 to the position depicted in Figure 7 might be conservative. The jet spectrum in epoch 4 is flat spectrum, $\alpha=0.41$. Thus, the jet might be optically thick. Therefore, due to SSA opacity, the true core position would be slightly upstream (to the left in Figure 7) from the epoch 4 core position. Extrapolating the shift by fitting the SSA core shift over frequency is logistically difficult. However, one can use the stationary secondary position as a nearby reference point in order to register the location of the core as a function of frequency in epoch 4. The first attempt did not work, because the $22.2 \mathrm{GHz}$ core position was very far from the power law fit to the other 3 points. We attribute this to the ejected component K3 that is unresolved in the $22.2 \mathrm{GHz}$ beam. Assuming a steep power law and a large SSA opacity at $15.3 \mathrm{GHz}$, we associated $5 \mathrm{mJy}$ at the position of $\mathrm{K} 3$ with the unresolved core at $22.2 \mathrm{GHz}$ and $\approx 0$ mJy in the unresolved core at $15.3 \mathrm{GHz}$ and $8.4 \mathrm{GHz}$. We re-register the $22.2 \mathrm{GHz}$ core position based on extricating the unresolved component, K3. The resultant powerlaw trend is plotted in the top frame of Figure 8 and extrapolated to high frequency. It is a plot that fits a core position in declination and right ascension with a power law for the associated core shift from this position as a function of frequency. This is an empirical process and the expectation is that the shift from the true core position is zero at high frequency where the SSA opacity is very small. The results are plotted in Figure 8 and indicates a 0.03 mas shift of the true core position based on SSA opacity effects. The top frame is the fit to the core 
shift and the bottom frame is a closeup of the core evolution and core shift resulting from this SSA opacity analysis. The SSA shifted core position in epoch 4 will be denoted as the AGN location.

Based on the shifted core position in Figure 8, we can estimate the velocity of the component K2. We know that component K2 was ejected after the flare start (after 2014 December 1, see Figures 1 and 2). It moved $0.40 \pm 0.04$ mas from the AGN in $<120$ days. Using a conversion of $1 \mathrm{mas}=0.8 \mathrm{pc}$, this equates to an apparent velocity $\beta_{\text {app }}=v_{\text {app }} / \mathrm{c}>$ $3.15 \pm 0.3$ for $\mathrm{K} 2$. This is a lower bound because there were likely numerous other mini-flares (associated with local maxima) during the rise of the light curve as discussed above. It is probably more likely that one of these mini-flares during the flare rise is associated with the ejection of the detected component $\mathrm{K} 2$. The $17.6 \mathrm{GHz}$ light curve seems to indicate that the 2015 radio flare in Mrk 231 was composed of multiple small superluminal ejections.

The component K3 is located 0.26 \pm 0.04 mas from the AGN. However, due to the poor beam shape in epochs 2 and 3, we have no reliable means of constraining the velocity of K3. Finally, we note that the trajectories for $\mathrm{K} 2\left(\mathrm{PA}=-117^{\circ}\right)$ and $\mathrm{K} 3\left(\mathrm{PA}=-137^{\circ}\right)$ appear to be different. K2 appears to be on a trajectory headed towards the steep spectrum secondary, K1. Component K3 curiously lies below a common line in Figure 7 that connects all the core positions and the discrete components, K1 and K2. Reprocessing the data multiple times consistently found that K3 lies to the south of this line.

\subsection{The Stationary Secondary}

If we assign the AGN location to the core at epoch 4 then as discussed above, the component K1 moved $<0.05$ mas in 9 years which is consistent with zero velocity with our level of measurement accuracy. However, if we converted this to a velocity it would equal $4200 \mathrm{~km} / \mathrm{s}$ which is too small for us to measure accurately. In Reynolds et al. (2009), we noted that matched (to $5 \mathrm{GHz}$ ) resolution VLBA observations indicated that the secondary is seen through a shroud of free-free absorbing gas with an emission measure of $\approx 10^{8} \mathrm{~cm}^{-6} \mathrm{pc}$. It was concluded that the steep spectrum secondary seems to be a radio lobe associated with the jet advancing into a dense medium (the jet is confined by ram pressure) that is also the source of the free-free absorption. It was estimated that the long term time averaged kinetic energy flux deposited in the stationary secondary is $\bar{Q} \approx 10^{42} \mathrm{ergs} \mathrm{s}^{-1}$.

Figure 9 compares the spectrum of component K1 in our four epochs of observation to

the historical spectra that were presented in Reynolds et al. (2009). The flux has approximately doubled in the last 9 years. This is consistent with the interpretation of K1 that it 
is a radio lobe confined by a dense medium. The light curve in Figures 1 and 2 indicates increased radio activity since 2011. The cumulative effect is to energize the radio lobe with more high energy plasma. There even appears to be an additional $>20 \%$ increase in the 8.4 GHz flux density during our observing program between epochs 1 and 3 according to Table 1 . This is apparently real since the secondary calibrator J1311+5513 does not show the same increase. If we associate the increase to plasma deposited in the lobe by the strong 2013 flare then according to Figure 1 the time of flight from the nucleus to K1 at epoch 3 is $1.7-2.5$ years. However, the flux increase at $8.4 \mathrm{GHz}$ is basically a linear rise from epoch 1 to epoch 3 (see Table 1). Thus, the time elapsed between the 2013 flare and epoch 1 is $\approx 1.5-2.3$ years. This time frame covers the rise of the 2013 flare to the small flare during the light curve decay of the 2013 flare. The peak of the 2013 flare was 1.9-2.0 years before the epoch 1 observation. The projected distance on the sky plane between the core and $\mathrm{K} 1$ is $3.2 \mathrm{lt}-\mathrm{yr}$. The apparent speed that the energy flux propagated with is $(3.2 \mathrm{lt}-\mathrm{yr} / 2.3$ $\mathrm{yr})<\beta_{\text {app }}<(3.2$ lt-yr $/ 1.5 \mathrm{yr})$, or $1.4<\beta_{\text {app }}<2.1$, consistent with mildly relativistic motion. In this scenario, based on the estimate of the velocity of the component, $\mathrm{K} 2$, in the last section this value decreases as the jet propagates toward K1. This can occur if the line of sight to the jet increased dramatically or there was deceleration of the flow. A change in the line of sight is inconsistent with the tight alignment of components and the core shifts with the direction from the core to K1 in Figure 7. This favors deceleration, which was suggested to occur by entrainment in the dense nuclear environment of Mrk 231 (Reynolds et al. 2009). Alternatively, if the increase of flux in $\mathrm{K} 1$ is from the 2015 flare, $\beta_{a p p}>9$. Neither scenario is ruled out by the data. Using the $\mathrm{K} 1$ radio lobe as a crude bolometer indicates that the jet in Mrk 231 is becoming more energetic. Mrk 231 might be transforming from a radio quiet quasar to a radio loud quasar.

\subsection{Summary of Sub-Parsec Scale Radio Structure}

In this section we used four epochs of VLBA observations in order to constrain the morphology and time evolution of the sub-parsec scale nuclear region during the major radio flare of 2015. The first result, in Section 3.1, was the detection of partially resolved

substructure at $43 \mathrm{GHz}$ in epochs 1 and 4 . We interpret these as ejecta associated with the major flare. The lack of the detection of substructure in epochs 2 and 3 is likely a consequence of a poorly shaped beam that reduced the resolution along the jet direction. This hindered our efforts to follow the time evolution of ejected components. In the absence of these data, we relied on determining a precise location of the actual AGN in order to constrain the jet speed in Section 3.2. This required a careful analysis of all four datasets. We determined a lower bound on the apparent speed of the ejection that was detected in 
epoch 1, > 3.15c. In Section 3.3, we showed that the stationary secondary (the putative radio lobe) doubled in $8.4 \mathrm{GHz}$ flux density over 9 years. We even detected a flux increase during this observing program. By associating this flux increase with energy from the 2013 radio flare, we estimate an average apparent jet speed of $1.4<\beta_{\text {app }}<2.1$. Alternatively, if the increase of flux in the stationary secondary is from the present flare, $\beta_{a p p}>9$. Neither scenario is ruled out by the data.

\section{The Far UV Jet Connection}

In this section, we explore the possible relationships between the far UV spectrum and the radio state of the nucleus.

\subsection{The Timing of the Flare Initiation and the HST COS G140L Observation}

The 2014 December 13 HST COS G140L observation was less than 36 hours before the first detected onset of flare activity on 2014 December 15 as evidenced by the $17.6 \mathrm{GHz}$ light curve in Figures 1 and 2. From 2014 November 15 until 2014 December 7 the $17.6 \mathrm{GHz}$ flux density was in a slow monotonic decline from $132 \mathrm{mJy}$ to $127 \mathrm{mJy}$. The next data point (MJD 57007) was 8 days later and the flux density increased to $142 \mathrm{mJy}$. This increase was not a statistical fluctuation or systematic observational error since the light curve showed steady growth afterwards (except for one outlier point in bad weather 29 days later). Furthermore, the spectral index from $14.6 \mathrm{GHz}$ to $17.6 \mathrm{GHz}$ showed a linear decrease (spectral flattening) from MJD 56985 until MJD 57019, going from $\alpha=1.1$ to $\alpha=0.3$ (see the top frame of Figure 10). The spectral index has a large uncertainty and using $14.6 \mathrm{GHz}$ for our low frequency instead of $13.8 \mathrm{GHz}$ is much more reliable with AMI data. However, there is a consistent trend of spectral hardening that is evident more than a week before the $17.6 \mathrm{GHz}$ flux density begins to increase and for 12 days afterwards.

The changes to the nucleus would have been profound because the preponderance of the $17.6 \mathrm{GHz}$ flux is from the stationary secondary and the surrounding large scale radio emission (Ulvestad et al. 1999a). We can estimate these two background components. In order to determine the flux density of the stationary secondary, $F_{\nu}^{S S}$, we note the increase of the flux density during the first three epochs of our VLBA program that was discussed in the last section. This increase was much larger at $8.4 \mathrm{GHz}$ than at $15.3 \mathrm{GHz}$. Thus, we expect a different time dependence for different frequencies. We estimate the flux densities at $14.6 \mathrm{GHz}$ and $17.6 \mathrm{GHz}$ in the four VLBA epochs by extrapolating the $15.3 \mathrm{GHz}$ flux 
density using the two point spectral indices to $8.4 \mathrm{GHz}$ and $22.2 \mathrm{GHz}$. We find at $14.6 \mathrm{GHz}$ that $F_{\nu}^{S S}(\nu=14.6 \mathrm{GHz})=99 \mathrm{mJy}, 104 \mathrm{mJy}$ and $111 \mathrm{mJy}$ in epochs 1,2 and 3, respectively. Similarly, we find at $17.6 \mathrm{GHz}$ that $F_{\nu}^{S S}(\nu=17.6) \mathrm{GHz}=74 \mathrm{mJy}, 80 \mathrm{mJy}$ and $83 \mathrm{mJy}$ in epochs 1, 2 and 3, respectively. We fit these data by powerlaws in time and extrapolate back to the time of the HST G140L observation

$$
F_{\nu}^{S S}(\nu=14.6 \mathrm{GHz})=85 \mathrm{mJy}, \mathrm{F}_{\nu}^{S S}(\nu=17.6 \mathrm{GHz})=67 \mathrm{mJy} \text { MJD } 57005 .
$$

We can find the background large scale flux density, $F_{L}^{L S}$, using the simultaneous VLA and VLBA observations in December 1996 (Ulvestad et al. 1999a). Being large scale in nature $(\sim 100 \mathrm{pc})$, these fluxes should be steady over 20 years. The VLBA images only the core and the stationary secondary with combined flux densities of $132 \mathrm{mJy}$ and $63 \mathrm{mJy}$ at $8.4 \mathrm{GHz}$ and $15.4 \mathrm{GHz}$, respectively. The VLA is sensitive to larger scale structures and detected flux densities of $203 \mathrm{mJy}$ and $112 \mathrm{mJy}$ at $8.4 \mathrm{GHz}$ and $15.4 \mathrm{GHz}$, respectively. Subtracting the VLBA flux from the VLA flux yields

$$
F_{\nu}^{L S}(\nu=8.4 \mathrm{GHz})=71 \mathrm{mJy}, \mathrm{F}_{\nu}^{\mathrm{LS}}(\nu=15.4 \mathrm{GHz})=49 \mathrm{mJy} .
$$

Fitting the data in Equation (2) by a power law with spectral index of $\alpha=0.62$, these flux densities can be used to extrapolate an estimate of the desired result,

$$
F_{\nu}^{L S}(\nu=14.6 \mathrm{GHz})=51 \mathrm{mJy}, \mathrm{F}_{\nu}^{\mathrm{LS}}(\nu=17.6 \mathrm{GHz})=45 \mathrm{mJy} .
$$

The core flux, $F_{\nu}^{\text {core }}$, can be estimated from the AMI measured flux density, $F_{\nu}$, by

$$
F_{\nu}^{\text {core }} \approx F_{\nu}-F_{\nu}^{L S}-F_{\nu}^{S S} .
$$

We can check Equation (4) during the first two epochs of VLBA observations at $17.6 \mathrm{GHz}$. Recall that $F_{\nu}^{S S}(\nu=17.6 \mathrm{GHz})=74 \mathrm{mJy}$ and $80 \mathrm{mJy}$ from above in epochs 1 and 2 . The extrapolated core flux is $F_{\nu}^{\text {core }}=90 \mathrm{mJy}$ in both epochs. Thus, using Equation (3), Equation $(4)$ predicts that $F_{\nu}(\nu=17.6 \mathrm{GHz}) \approx 45+90+74 \mathrm{mJy}=209 \mathrm{mJy}$ in epoch 1 and $F_{\nu}(\nu=17.6 \mathrm{GHz}) \approx 45+90+80 \mathrm{mJy}=215 \mathrm{mJy}$ in epoch 2 which is consistent with the levels measured (quasi-simultaneously) during plateau 1 in Figure 6. With the confidence provided by this consistency check, we estimate $F_{\nu}^{\text {core }}$ from the AMI light curve in the bottom frame of Figure 10. Note that the estimated $F_{\nu}(\nu=17.6 \mathrm{GHz})$ increases from $14 \mathrm{mJy}$ to $30 \mathrm{mJy}$ at the time of the HST observation and the spectral index is extremely inverted and a short extrapolation to $22.2 \mathrm{GHz}$ implies that $F_{\nu}(\nu=22.2 \mathrm{GHz}) \approx 50 \mathrm{mJy}$. The bottom frame of Figure 10 indicates that the nuclear spectrum hardens drastically a few days to 2 weeks before the G140L observation. A hardening of the spectrum is expected before the rise of the low frequency flux in the early stages of an optically thick flare. The implication 
is that the flux was increasing at high frequency during this time period. As the ejected plasma propagates to larger dimension, the SSA opacity decreases. Around the time of the G140L observations, the spectrum begins to soften and the SSA opacity is sufficiently low at $17.6 \mathrm{GHz}$ that the flux density starts to increase rapidly: increasing by a factor of 4 in less than three weeks.

The conclusion is that the elevated flux on 2014 December 15 (MJD 57005.5) is a direct consequence of the flare that peaked 6 months later. Because of the gap in observations between 2014 December 8 and 2014 December 15, the light curve could have begun a drastic increase at any time in between. Furthermore, for an optically thick flare at $17.6 \mathrm{GHz}$, the $43 \mathrm{GHz}$ flux density increase leads the increase of the $17.6 \mathrm{GHz}$ flux density. Thus, the 2014 December 13 HST COS G140L observation was most likely a few days to two weeks after the flare initiated.

\subsection{Previous HST Observations}

Another observation that occurred during an active radio state was the HST COS G130M observation on 2011 October 15 that was indicated in the top frame of Figure 1. Figure 11 is a magnified view of the radio flare so that one can see the radio state at the time of the HST observation. Note that the observation was during a very high $22 \mathrm{GHz}$ flux state. The flare was experiencing a saddle point that was either near the peak of the flare or on the declining side. It appears that both the G140L and the G130M observations occurred during very active jet states, at the start of a strong flare and during the last local maximum of another strong flare, respectively. Conversely, as discussed in terms of Figure 1, the 1996 November 21 HST FOS spectrum was taken near (within two weeks of) an historic low in the $22 \mathrm{GHz}$ radio flux.

There is a great disparity between the quality of the FOS and COS data-sets. The highest quality data are the G130M data from 2011. It is averaged over 10 observations that exceed a total of $12000 \mathrm{~s}$. These data were previously discussed in Veilleux, Trippe and Hamann et al. (2013); Veilleux. Melendez and Tripp et al. (2016) and the averaged data was generously provided by S. Veilleux and M. Melendez. However, these data had a rather narrow wavelength coverage. The G140L spectrum from 2014 was downloaded from the Milkulski Archive for the Space Telescope (MAST) and is of comparable quality with over 15,000 seconds of observation, but a much wider wavelength range and much lower spectral resolution. These data have been considered extensively in Veilleux. Melendez and Tripp et al. (2016). The FOS G160L data was a short 770s exposure with low resolution. The following discussion is highly dependent on this brief observation. The important aspect for this discussion is know- 
ing if the absolute flux calibration was accurate. In order to remove ambiguity, we excised the spectrum below $1315 \AA$, in order to avoid Geo OI coronal emission and Geo Ly $\alpha$ emission that is impossible to reliably extricate with the low resolution combined with the noisy edge of the spectral coverage. With these issues removed, we can assess the flux calibration. The observation was Post-COSTAR and the acquisition method was ACQ/PEAK that creates a centering uncertainty of 0.05" (Evans and Koratkar 2004). This equates to a 1\% flux uncertainty in the 1.0" circular aperture (FOS Instrument Science Reports CAL/FOS-140) that was used. The data acquisition files had no flags or warnings, so every indication is that the flux uncertainty should be $<10 \%$ (Evans and Koratkar 2004). The absolute flux density of the COS observations is likely better. The 10 different observations with G130M agree within $<5 \%$ in the overlapping ranges of wavelength, similarly for the two observations with G140L.

\subsection{Comparing the Three Epochs of HST Observations}

The far UV spectra are compared in Figure 12 by plotting each pair of spectral energy distributions (SEDs) in different panels. Plotting all three at once is too cluttered for comparative purposes. There are some amazing similarities and striking contrasts that are catalogued in Table 2. The top frame in Figure 12 compares the two SEDs that were sampled when the radio jet was in a state of high activity. There is no observable difference in the region of overlap between $1150 \AA$ and $1470 \AA$. The Geo OI coronal emission and Geo Ly $\alpha$ emission were left in for reference in the higher spectral resolution G130M SED. These regions were excised from the G140L SED for clarity. The spectral index of $\alpha_{\lambda}=1.7$ in column (8) of Table 2 for the continuum flux density, $F_{\lambda} \sim \lambda^{\alpha}$, between $1315 \AA$ and $1050 \AA$ agrees with the value found previously for the G130M spectrum (Veilleux, Trippe and Hamann et al. 2013). Formally, the spectral coverage ends at $1150 \AA$, so the last $100 \AA$ is an extrapolation. The G140L spectrum shows a spectral break near $1315 \AA$.

The middle frame compares the high activity jet state at the beginning of the 2015 flare and the 1996 low radio luminosity state. The SED is very similar from $1400 \AA$ to $1900 \AA$, even after 18 years. However shortward of $1400 \AA$ there is a clear visual excess in the 1996 SED. We would like to quantify what we see visually. One method was to compute the integrated flux in two bands, $F(1365 \pm 50 \AA)$ in column (6) of Table 2 and in the range $F(1850 \pm 50 \AA)$ in column (7) of Table 2. Even though the flux in the band $F(1850 \pm 50 \AA)$ is slightly larger in 2014, it is significantly smaller in the band $F(1365 \pm 50 \AA)$ in 2014 . Visually it is very clear in the figure that the G140L SED angles downward toward shorter wavelength between $1315 \AA$ and $1900 \AA$. However, the data are inconsistent with such a downward slant in 1996 . 


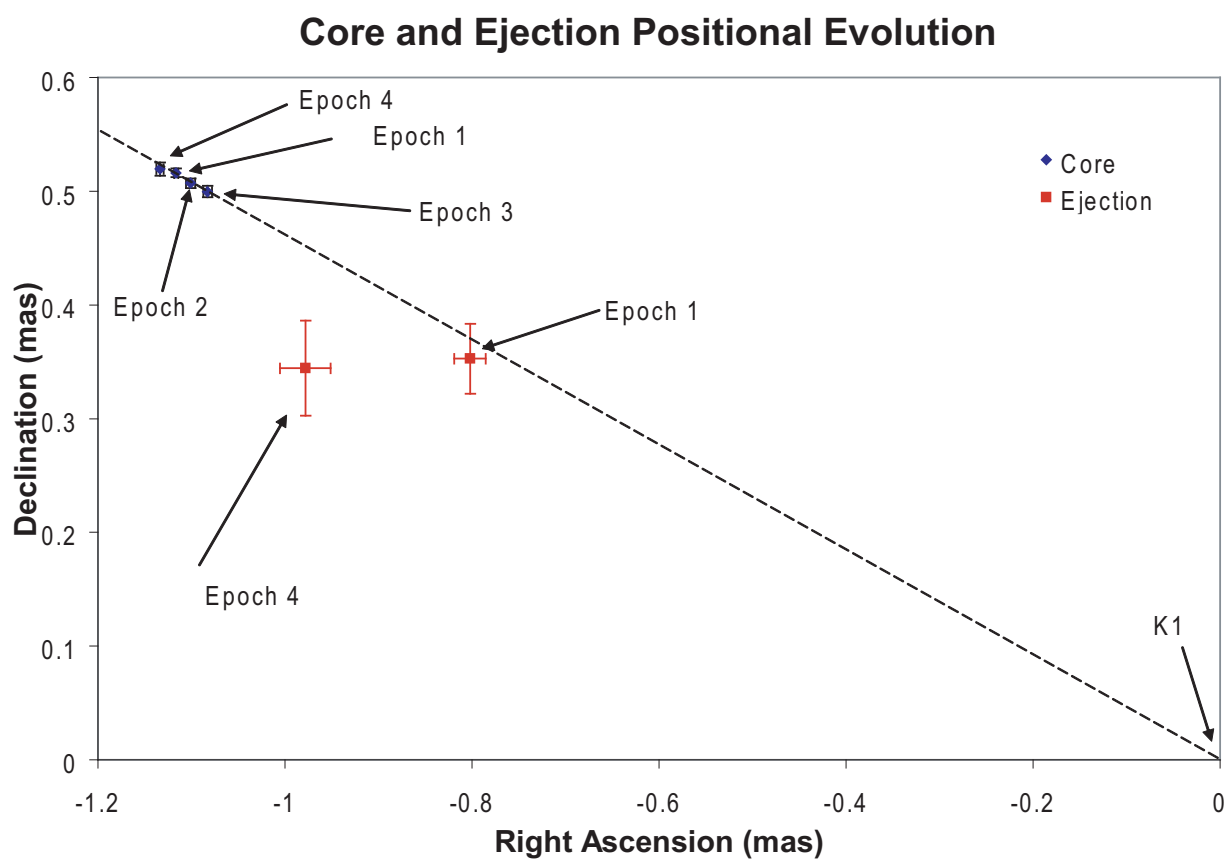

Fig. 7.- The position of the core and the ejection relative to the astrometric origin K1.

Table 2: Far UV Spectral Properties Versus High Frequency Radio Properties

\begin{tabular}{|c|c|c|c|c|c|c|c|c|c|}
\hline $\begin{array}{c}1 \\
\text { Date }\end{array}$ & $\begin{array}{c}2 \\
17.6 \mathrm{GHz}\end{array}$ & $\begin{array}{c}3 \\
22 \mathrm{GHz}\end{array}$ & $\begin{array}{l}4 \\
\text { Date }\end{array}$ & $\begin{array}{c}5 \\
\text { Grating }\end{array}$ & $\begin{array}{c}6 \\
F(1365 \pm 50 \AA)\end{array}$ & $\begin{array}{c}7 \\
F(1850 \pm 50 \AA)\end{array}$ & $\begin{array}{c}8 \\
\alpha_{1}\end{array}$ & $\begin{array}{c}9 \mathrm{~b} \\
\alpha_{1}\end{array}$ & $\begin{array}{c}10 \\
\text { BALNIcity }\end{array}$ \\
\hline Date & $\begin{array}{l}17.6 \mathrm{GHz} \\
\text { Flux }\end{array}$ & $\begin{array}{l}22 \mathrm{GHz} \\
\text { Flux }\end{array}$ & & & & & & & \\
\hline $\begin{array}{l}\text { Radio } \\
\text { Obs. }\end{array}$ & $\begin{array}{l}\text { Density } \\
(\mathrm{mJy})\end{array}$ & $\begin{array}{c}\text { Density } \\
(\mathrm{mJy})\end{array}$ & $\begin{array}{l}\text { HST } \\
\text { Obs. }\end{array}$ & $\begin{array}{l}\text { Spectro- } \\
\text { graph }\end{array}$ & $\begin{array}{l}10^{-15} \\
\operatorname{ergs~s}^{-1} \mathrm{~cm}^{-2}\end{array}$ & $\begin{array}{l}10^{-15} \\
\operatorname{ergs~s}^{-1} \mathrm{~cm}^{-2}\end{array}$ & & & $\begin{array}{c}\mathrm{CIV} \\
(\mathrm{km} / \mathrm{s})\end{array}$ \\
\hline 1996 Dec 05 & $\overline{90 \pm 10^{\mathrm{c}}}$ & $\overline{\overline{62 \pm 9}}$ & $211 / 21 / 1996$ & 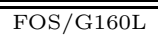 & $\overline{168 \pm 17}$ & $\overline{128 \pm 13}$ & & $\overline{\approx \approx-1}$ & $\overline{2 \approx 600^{\mathrm{d}}}$ \\
\hline 2011 Sep 30 & $\ldots$ & $200 \pm 10$ & 2011 Oct 15 & $\mathrm{COS} / \mathrm{G} 130 \mathrm{M}$ & $139 \pm 7$ & $\ldots$ & 1.7 & $\ldots$ & $\cdots$ \\
\hline 2011 Oct 25 & . & $180 \pm 5$ & 2011 Oct 15 & $\mathrm{COS} / \mathrm{G} 130 \mathrm{M}$ & $139 \pm 7$ & & 1.7 & 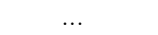 & \\
\hline 2014 Dec 15 & $142 \pm 5$ & $\ldots$ & 2014 Dec 13 & $\mathrm{COS} / \mathrm{G} 140 \mathrm{~L}$ & $140 \pm 7$ & $135 \pm 7$ & 1.5 & -0.1 & $0^{\mathrm{e}}$ \\
\hline
\end{tabular}

${ }^{a}$ Spectral index of continuum power law, $F_{\lambda} \sim \lambda^{\alpha}$. Computed from $1050 \AA$ to $1300 \AA$.

${ }^{a}$ Spectral index of continuum power law, $F_{\lambda} \sim \lambda^{\alpha}$. Computed from $1315 \AA$ to $1900 \AA$.

${ }^{c}$ estimated from the VLA data in Ulvestad et al. (1999a)

Gallagher et al. (2002)

Eeilleux, Melendez and Tripp et al. (2016) 


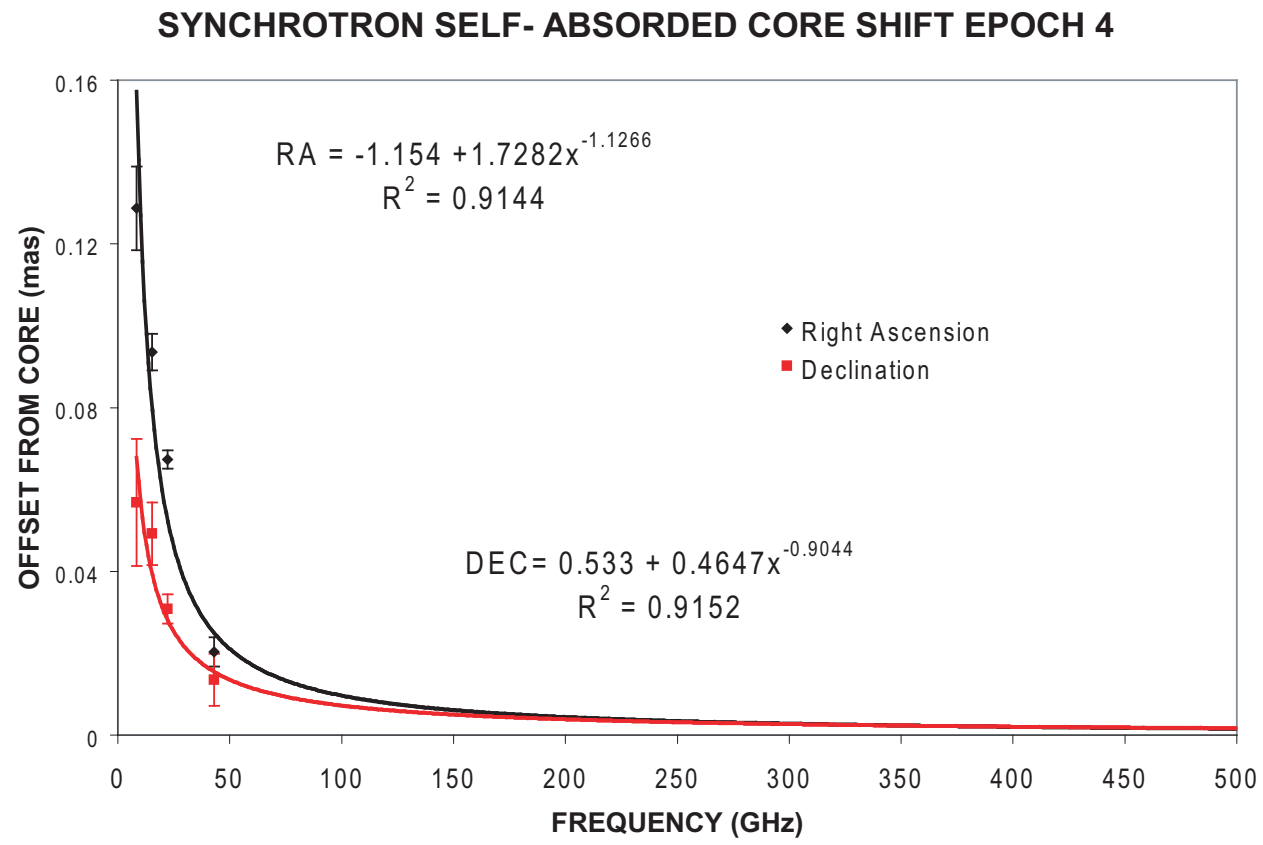

Core Positional Evolution and Core Shift

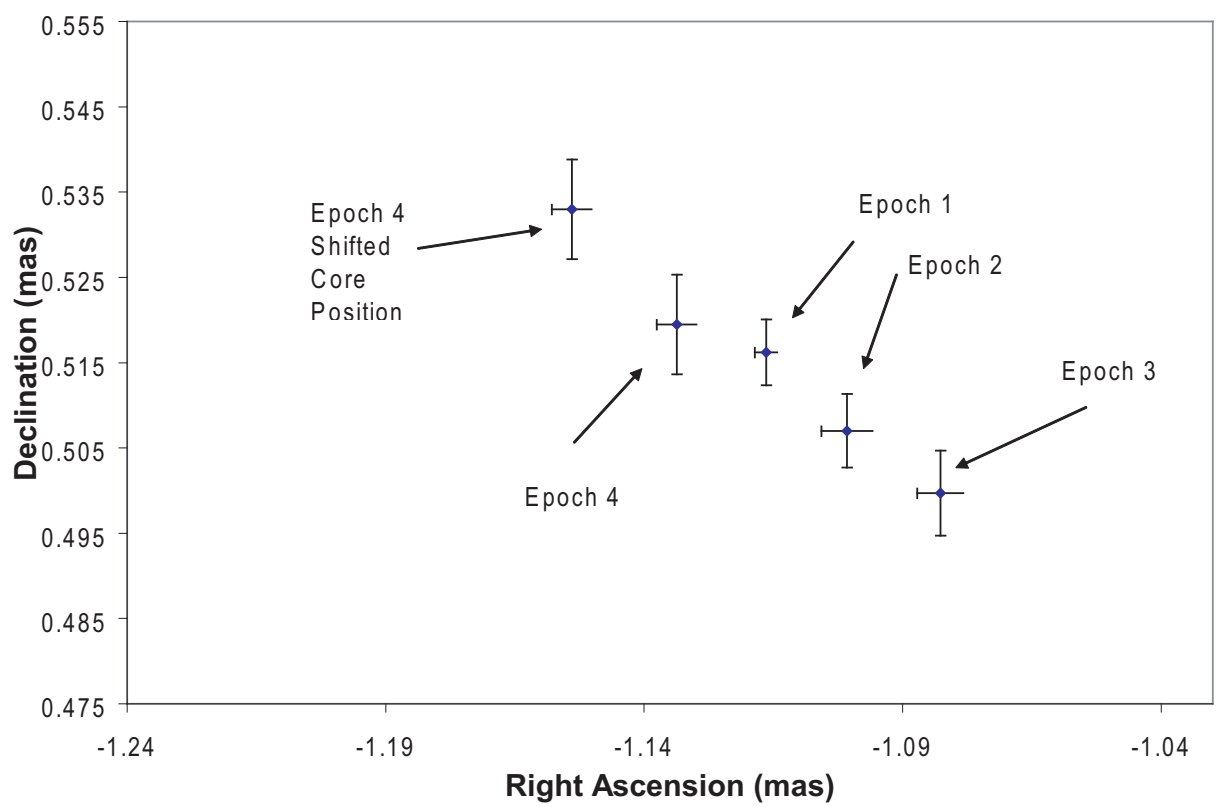

Fig. 8. - The top frame shows the shift of the core as a function of frequency in epoch 4 due to SSA opacity effects. The fits of the power laws to the data are good as evidenced by the large squared multiple regression values, $R^{2}$. The quantity, $\mathrm{x}$, in the equation for the core shift is the observed frequency in GHz. The bottom frame shows the shifted true core location that is consistent with zero offest at high frequency in the top frame. 


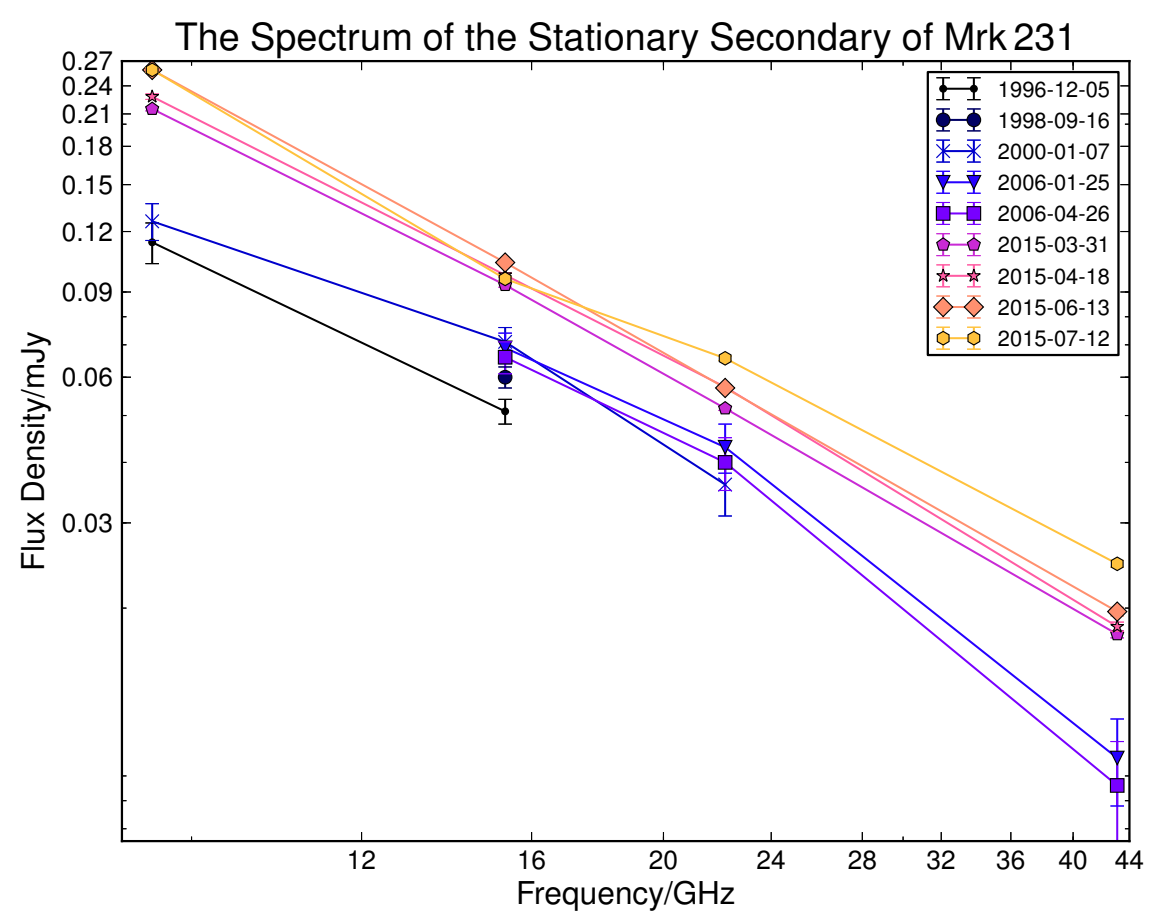

Fig. 9. - The historic spectrum of the stationary component K1. 

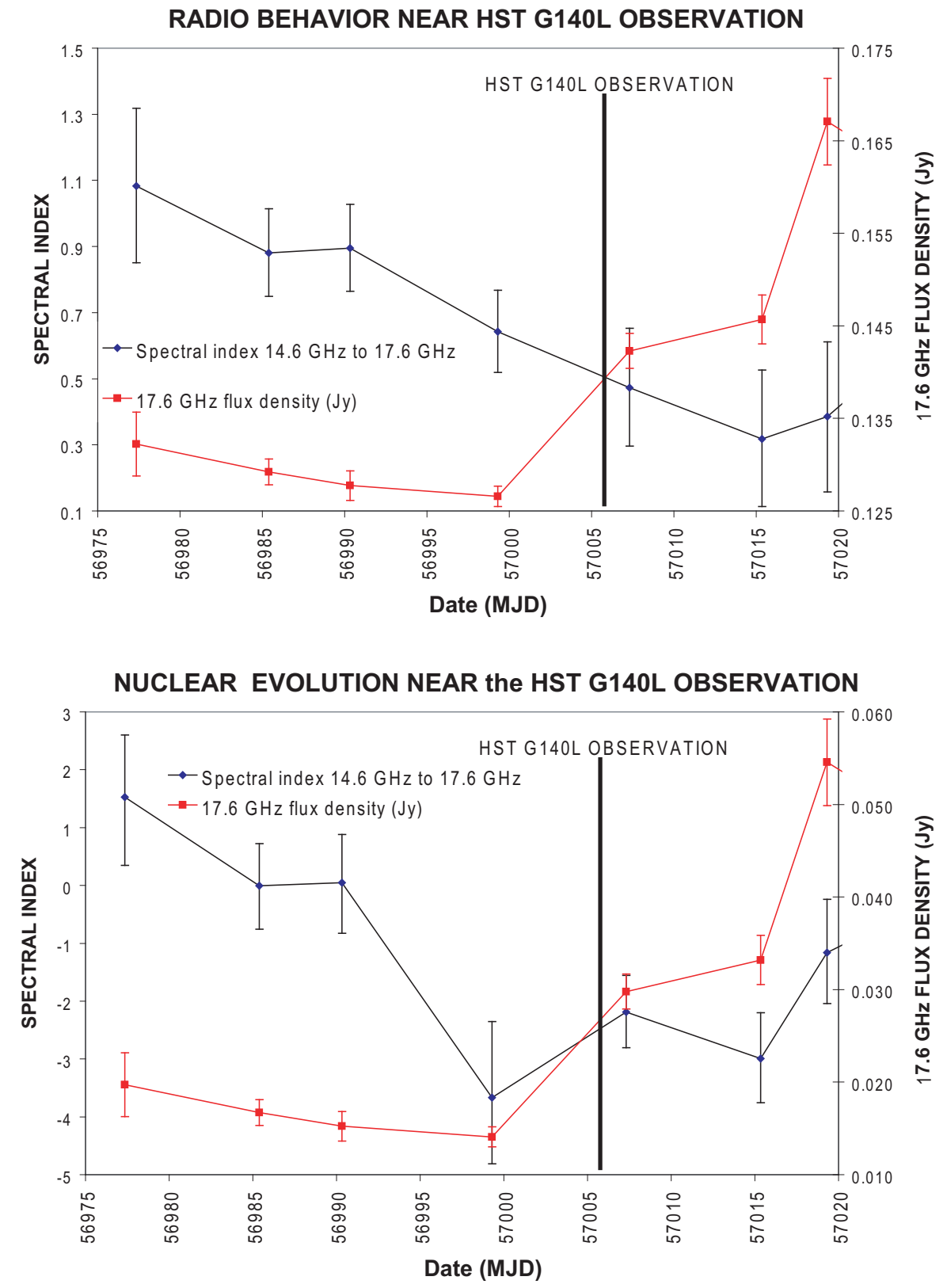

Fig. 10. - The beginning of the major radio flare that peaked in the spring of 2015. The top frame plots the total flux density from the AMI data. The spectral index has a large uncertainty. However, there is a consistent trend of spectral hardening that is evident more than a week before the $17.6 \mathrm{GHz}$ flux density begins to increase and for 12 days afterwards. The bottom frame describes the implied change to the nuclear component using Equations $(1)$ - (4) in the text. The spectrum hardens drastically a few days days to 2 weeks before the G140L observation. The implication is that the flux was increasing at high frequency during this time period. As the disturbance propagates away from the nucleus, the SSA opacity decreases. Around the time of the G140L observations, the spectrum begins to soften and the $17.6 \mathrm{GHz}$ flux density starts to increase rapidly: increasing by a factor of 4 in less than three weeks. 


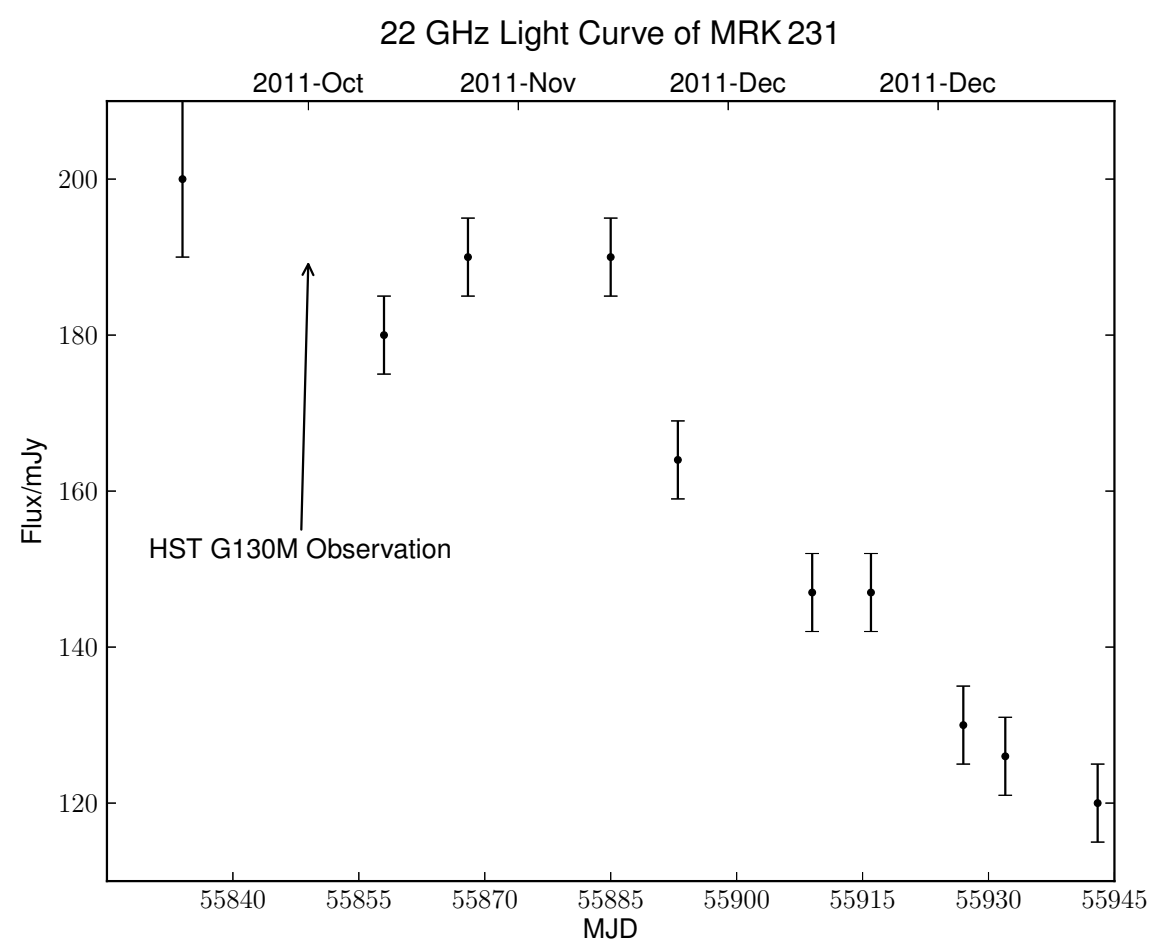

Fig. 11. - The HST COS G130M observation during the 2011 radio flare. 

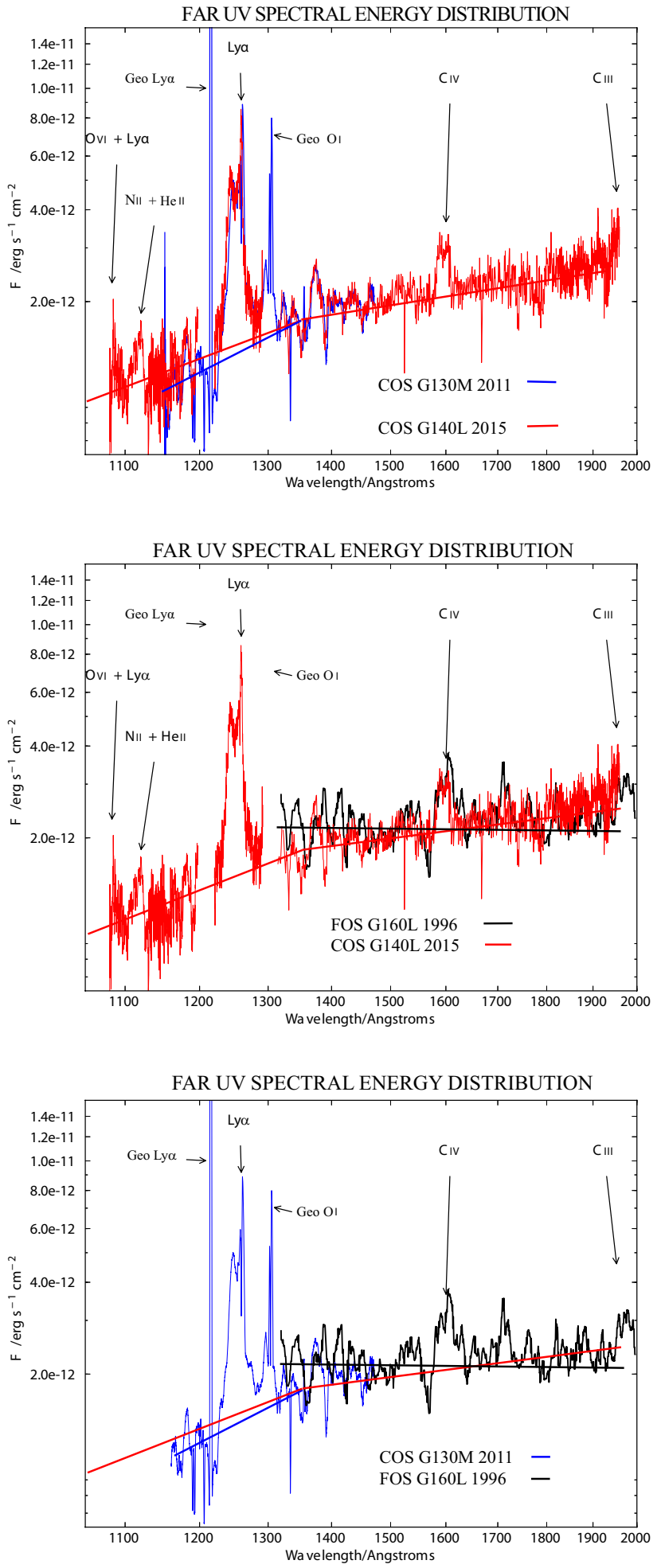

Fig. 12.- The top panel compares the SEDs of the two high radio states, the 2011 flare and the 2015 flare. The middle panel compares the SED during the 2015 flare and during the radio low state in 1996. There is CIV BAL absorption and a harder spectrum in 1996. The last frame compares the SED in 1996 and during the radio flare in 2013. The SEDs diverge shortward of $1400 \AA$. 
We quantify this in Table 2 in Column (9) with the spectral index $\alpha_{\lambda} \approx-1$ in 1996 (a flat SED) compared to $\alpha_{\lambda} \approx-0.1$ in 2014 . The other significant distinction is the depth of the CIV BAL trough. The BALnicity index, BI, was defined in Weymann et al. (1991) as

$$
B I=\int_{v=-25000}^{v=-3000}[1-F(v) / 0.9] C d v,
$$

where $F(v)$ is the flux density normalized to the continuum level as a function of $v$, the velocity from the QSO rest frame line emission frequency in $\mathrm{km} / \mathrm{s}$. The step function, $C(v) \neq 0$ if and only if there is more than $2000 \mathrm{~km} / \mathrm{s}$ of continuous absorption beyond -3000 $\mathrm{km} / \mathrm{s}$. This measure of broad absorption has been borne out over the years as very robust. More lax measures of absorption (such as associated absorption and mini-BALs) have turned out to represent different classes of objects, that have less X-ray absorption than BALQSOs and larger radio luminosity than bona-fide BALQSOs (Shankar et al. 2008; Punsly 2006; Knigge et al. 2008). Column (10) of Table 2 lists the estimated values of BI. During the low radio state in $1996, B I \approx 600 \mathrm{~km} / \mathrm{s}$ (Gallagher et al. 2002). At the beginning of the 2015 flare $B I=0 \mathrm{~km} / \mathrm{s}$ (Veilleux. Melendez and Tripp et al. 2016). In the middle panel of Figure 12 , it is evident that there is more high velocity absorption in 1996.

The bottom panel of Figure 12 compares the SED during the 2011 flare and the quiescent state in 1996. Even though the overlap in spectra is only from $1315 \AA$ to $1470 \AA$, it is clear that the SEDs agree in the range of $1400 \AA-1470 \AA$, but they clearly diverge shortward of this. The long wavelength end of the G140L continuum fit is left on the plot for an additional reference level.

In summary, based on limited data and the inability to secure an HST spectra during the peak of the 2015 as a target of opportunity, we find some trends that would need to be verified with more spectra.

1. The far UV emission longward of $1400 \AA$ is incredibly constant over an 18 year period. This finding is unusual for a quasar. Studies of far UV variability of quasars indicate measurable variability is the norm for these time frames although no variability is occasionally found (Macleod et al. 2012; Welsh et al. 2011; Wilhite et al. 2005).

2. There is a far UV flux deficit shortward of $1400 \AA$ during periods of increased relativistic jet activity, relative to the far UV flux in periods of low radio activity.

3. The high ionization BAL absorption is suppressed during periods of increased relativistic jet activity.

4. There is a sharp spectral break near $1400 \AA$ during periods of high jet activity. It is unknown what happens during periods of low radio emission. The peak of the far UV 


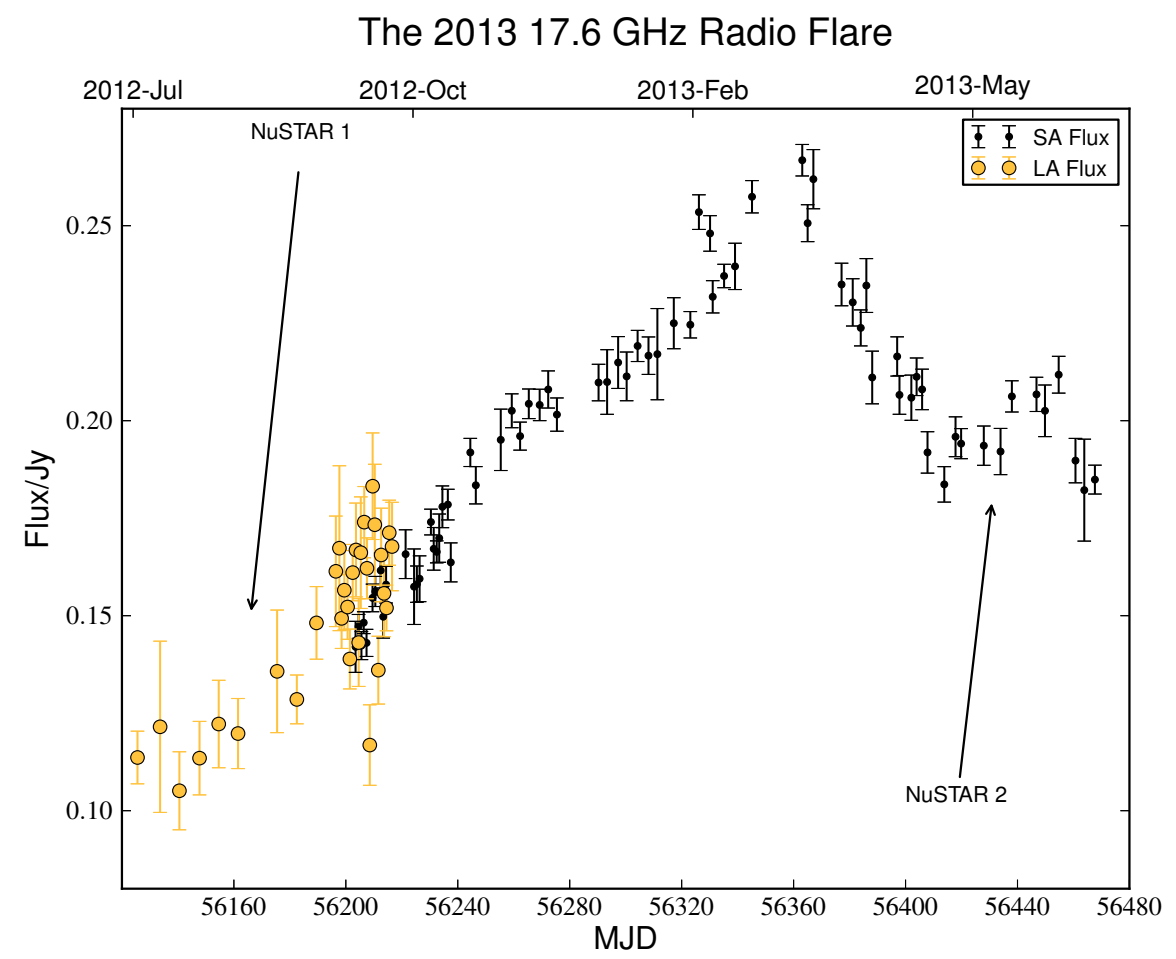

Fig. 13. - The light curve of the 2013 flare shows that the NuSTAR 1 observation occurred during a low radio state and the NuSTAR 2 observation occurred during a high radio state, As discussed in Reynolds et al. (2013b), the AMI monitoring began in the large array. However, as can be seen from the onset of some large scatter, calibration issues developed and we shifted to the small array. 
SED is longward of $1400 \AA$. The steep spectral index shortward of $1400 \AA, \alpha_{\lambda}=1.5-1.7$, is extremely steep and unusual for a quasar (Telfer et al. 2002).

\section{X-ray Observations}

Mrk 231 was observed three times with NuSTAR and twice with XMM-Newton during the 2015 campaign. As reported in Table 3, NuSTAR observed the source on April $2(\sim 32 \mathrm{ks}$ of net exposure), on April $19(\sim 28 \mathrm{ks})$, and on May $28(\sim 30 \mathrm{ks})$, while the two XMMNewton observations were carried out on April 25 ( $\sim 19 \mathrm{ks})$, and on May $28(\sim 21 \mathrm{ks})$. Table 3 also reports one previous XMM-Newton observation (performed on 2001 June 7) and two archival NuSTAR observations performed on 2012 August 26 and 2013 May 9 that we use for comparison purposes.

\subsection{The 2015 NUSTAR Observations}

In order to assess the relevance of the five pointed observations of Mrk 231 with NuSTAR in the context of jet activity, we consult the light curve in Figures 1 and 2. More resolution is needed in Figure 1 in order to clarify the radio state for the NuSTAR 1 observation. The light curve in Figure 13 of the 2013 flare indicates that the NuSTAR 1 observation occurred during a low radio state and the NuSTAR 2 observation occurred during a high radio state (as did the NuSTAR 3,4, and 5 observations in 2015). Thus, NuSTAR 1 is in a low radio state and NuSTAR 2-5 are all in a high radio state.

The NuSTAR 1 low radio state X-ray spectrum is compared with all other high radio flux NuSTAR observations in Figure 14. We note that the NuSTAR 1 observation presents a clear deficit of X-ray photons below $12 \mathrm{keV}$ with respect to the NuSTAR 2, 3 and 5 observations. This is especially noticeable bluewards of the Fe emission line (around $6 \mathrm{keV}$

in the observed frame) where there are hints for absorption structures in NuSTAR 1 between $6.5 \mathrm{keV}$ and $10 \mathrm{keV}$. The NuSTASR 1 and NuSTAR 4 spectra seem to be more similar, although some subtle difference may be present in two narrow bands areound $7 \mathrm{keV}$ and $8.5 \mathrm{keV}$. On the other hand, the high-energy spectra above $\sim 12 \mathrm{keV}$ look the same at all epochs. These qualitative claims are confirmed by comparing the (model-independent) background-subtracted count rates in the soft and hard bands for all observations. The net count rates are reported in Table 4, and they confirm that the NuSTAR 1 observation is characterized by a deficit of soft X-ray photons with respect to the NuSTAR 2, 3, and 5 pointings. NuSTAR 4 appears to have a soft X-ray deficit similar to NuSTAR 1, while high- 
energy count rates above $12 \mathrm{keV}$ are consistent with each other at all epochs, with perhaps a hint of decreased flux in the last NuSTAR observation. This suggests that any difference in the X-ray spectrum between epochs is likely due to intervening absorption rather than being intrinsic. The shape of the NUSTAR 1 spectrum between $6.5 \mathrm{keV}$ and $10 \mathrm{keV}$ seems indeed to suggests that some absorption feature is present in that band. This structure may be present in other observations as well, but with reduced strength.

We then proceed by fitting all available NuSTAR data in the highest signal-to-noise band, namely the $3-30 \mathrm{keV}$ one. Mrk 231 is unresolved by the NuSTAR beam, thus star formation and high mass X-ray binary emission are responsible for a non-negligible fraction of the total X-ray emission detected by NuSTAR (Teng et al. 2014). We do not want to speculate on complicated models with many uncertainties and assumptions and we use here some very simple spectral models. The baseline model is that of a simple power law continuum absorbed by a Galactic column density fixed at $9.64 \times 10^{19} \mathrm{~cm}^{-2}$, Kalberla et al. (2005)) plus an extra absorbing column of neutral gas at the redshift of the source, and a Gaussian emission line with $\sigma$ fixed to $10 \mathrm{eV}$ (unresolved at the resolution of the NuSTAR A and B detectors) to reproduce the Fe K emission line (the line energy is restricted between $6.4 \mathrm{keV}$ and $6.97 \mathrm{keV}$ in the rest-frame). All our spectral analysis is first carried out considering the FPM A and B NuSTAR detectors separately, including a cross-normalization constant between the two. We then produce co-added spectra $(\mathrm{A}+\mathrm{B})$ for all observations, and we re-fit the co-added spectra with the same spectral model. As we do not find any statistically significant difference in any of the spectral parameters we derive, we proceed by reporting results for the co-added $\mathrm{A}+\mathrm{B}$ spectra.

All of the NuSTAR observations except for the low radio state NuSTAR 1 observation are well fitted by the simple baseline model with $\chi^{2}$ of the order of unity. NuSTAR 1 represents the exception, and the baseline model provides a poor fit to the data with $\chi^{2}=82$ for 56 degrees of freedom (dof). The second worse fit is obtained for NuSTAR 4 (as expected given that this is the observation that mostly resembles NuSTAR 1, see Figure 14) where we have $\chi^{2}=58$ for 40 dof. Our results are reported in Table 5, and the peculiar NuSTAR 1 spectrum and best-fitting baseline model are shown in the left panel of Figure 15.

It is interesting to note that the only data set that is not well described by the baseline model (the NuSTAR 1 low radio state spectrum in 2012) was used, together with a simultaneous and higher quality Chandra X-ray spectrum (and to the NuSTAR 2 data) to argue that an ultra-fast wind is present in Mrk231. The evidence for such wind was detected in X-ray absorption from $6.5 \mathrm{keV}$ to $10 \mathrm{keV}$ (Feruglio et al. 2015). This appears to be a likely explanation for the deficit of X-ray photons in the 6.5-10 keV band during the NuSTAR 1 observation (see the left panel of Figure 15) although our analysis seems to exclude that the 
Table 3: Log of the X-ray observations used in this work.

\begin{tabular}{lcccc}
\hline \hline Mission & Name & Date & Detector & Net exposure \\
\hline \hline XMM-Newton & XMM 1 & 2001 Jun 07 & EPIC-pn & $17 \mathrm{ks}$ \\
\hline NuSTAR & NuSTAR 1 & 2012 Aug 27 & FPM (A-B) & $41 \mathrm{ks}$ \\
\hline NuSTAR & NuSTAR 2 & 2013 May 09 & FPM (A-B) & $29 \mathrm{ks}$ \\
\hline \multicolumn{5}{c}{} \\
\hline NuSTAR & NuSTAR 3 & 2015 Apr 02 & FPM (A-B) & $32 \mathrm{ks}$ \\
\hline NuSTAR & NuSTAR 4 & 2015 Apr 19 & FPM (A-B) & $28 \mathrm{ks}$ \\
\hline XMM-Newton & XMM 2 & 2015 Apr 25 & EPIC-pn & $19 \mathrm{ks}$ \\
\hline NuSTAR & NuSTAR 5 & 2015 May 28 & FPM (A-B) & $30 \mathrm{ks}$ \\
\hline XMM-Newton & XMM 3 & 2015 May 28 & EPIC-pn & $21 \mathrm{ks}$ \\
\hline \hline
\end{tabular}

Table 4: The net count rates for all NuSTAR observations in the 3-12 keV and 12-30 keV bands. Count rates are derived from the co-added $\mathrm{A}+\mathrm{B}$ detectors data for all observations. Units are $10^{-2} \operatorname{cts~s}^{-1}$.

\begin{tabular}{lcc}
\hline \hline Observation & 3-12 keV count rate & $12-30 \mathrm{keV}$ count rate \\
\hline \hline NuSTAR 1 & $1.44 \pm 0.05$ & $0.56 \pm 0.03$ \\
NuSTAR 2 & $1.89 \pm 0.06$ & $0.61 \pm 0.04$ \\
NuSTAR 3 & $1.97 \pm 0.06$ & $0.57 \pm 0.03$ \\
NuSTAR 4 & $1.62 \pm 0.06$ & $0.54 \pm 0.04$ \\
NuSTAR 5 & $1.79 \pm 0.06$ & $0.52 \pm 0.03$ \\
\hline \hline
\end{tabular}



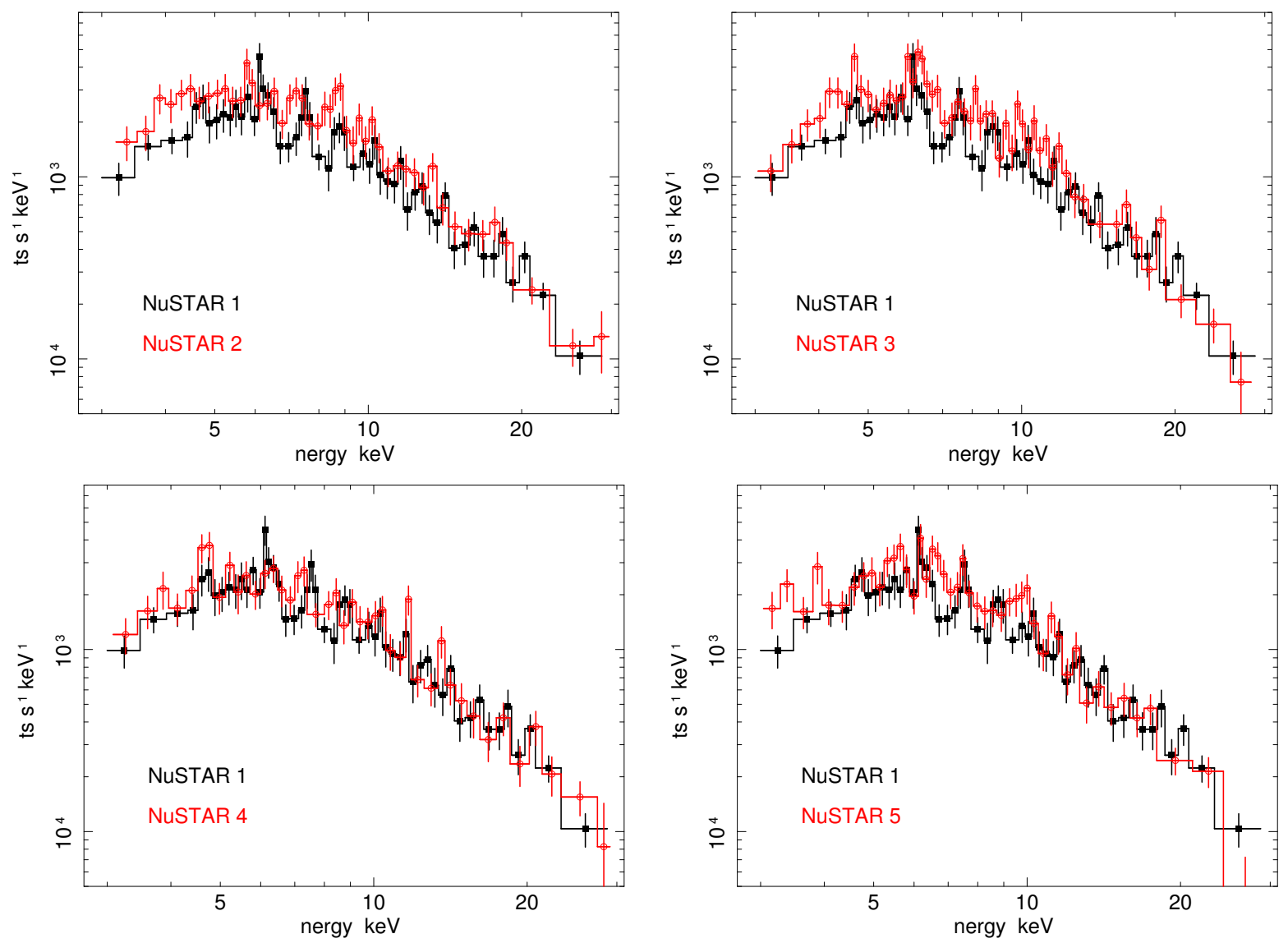

Fig. 14. - The low radio flux state NuSTAR 1 X-ray spectrum (black filled squares in all panels) is compared with all other high radio flux NuSTAR spectra (red empty circles in all panels) in the most sensitive NuSTAR band (3-30 keV). Each spectrum is the result of coadding the FPM A and B NuSTAR detectors, as this improves visual clarity. The co-added spectra are produced by combining source and background spectra, as well as responses using the addspec FTOOL available within the HEASOFT package. Solid lines are plotted only to guide the eye, and they step through all data points (no spectral fit is included here). 
Table 5: Baseline model fits to all NuSTAR observations. Column densities are expressed in units of $10^{22} \mathrm{~cm}^{-2}$, the $\mathrm{Fe}$ line energy is in $\mathrm{keV}$, and its intensity is in units of $10^{-6} \mathrm{ph} \mathrm{s}^{-1} \mathrm{~cm}^{-2}$. Note that the Fe line is not formally detected during the NuSTAR 2 and 4 observations (we fix its energy to $6.6 \mathrm{keV}$ in order to compute an upper limit on its intensity).

\begin{tabular}{lccccc}
\hline \hline Observation & $\Gamma$ & $N_{\mathrm{H}}$ & $E_{\mathrm{Fe}}$ & $I_{\mathrm{Fe}}$ & $\chi^{2} / \mathrm{dof}$ \\
NuSTAR 1 & $1.20 \pm 0.20$ & $5 \pm 4$ & $6.5 \pm 0.1$ & $3.5 \pm 2.0$ & $82 / 56$ \\
NuSTAR 2 & $1.50 \pm 0.20$ & $5 \pm 4$ & $6.6^{f}$ & $\leq 2.5$ & $47 / 45$ \\
NuSTAR 3 & $1.60 \pm 0.20$ & $10 \pm 5$ & $6.55 \pm 0.15$ & $4.4 \pm 2.3$ & $54 / 52$ \\
NuSTAR 4 & $1.40 \pm 0.20$ & $5 \pm 4$ & $6.6^{f}$ & $\leq 3.5$ & $58 / 40$ \\
NuSTAR 5 & $1.50 \pm 0.20$ & $5 \pm 4$ & $6.7 \pm 0.2$ & $2.8 \pm 1.8$ & $54 / 45$ \\
\hline \hline
\end{tabular}
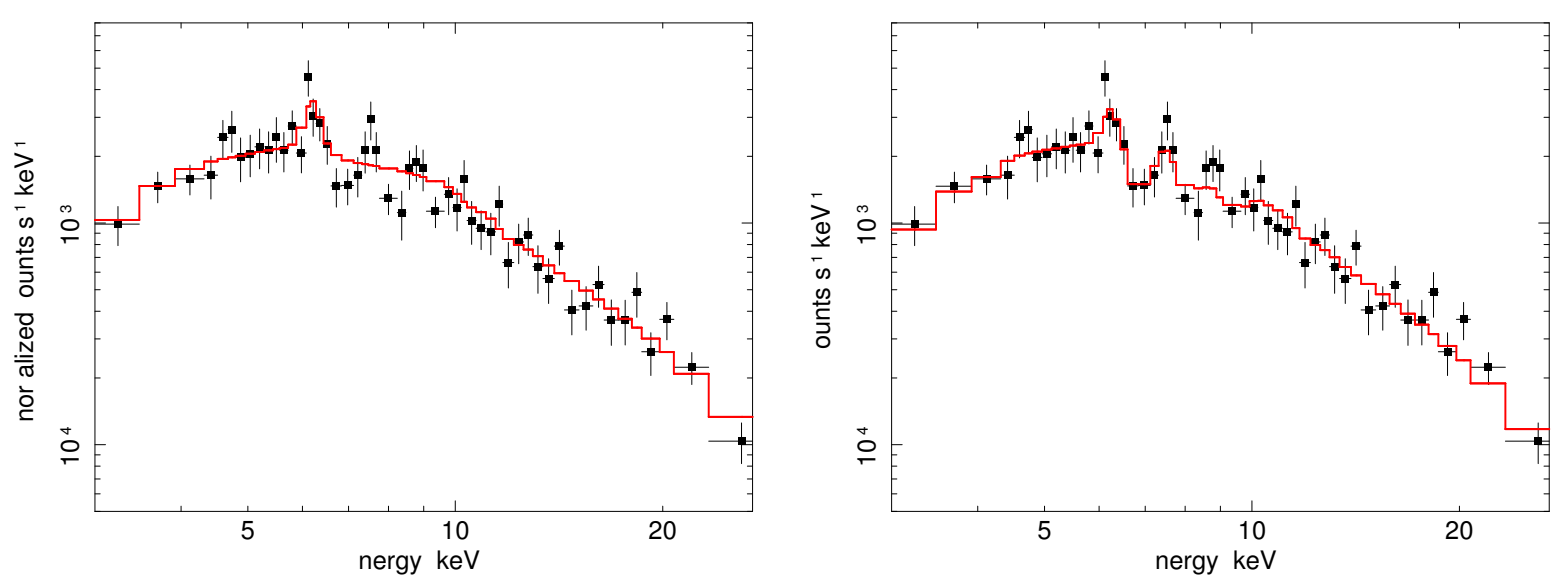

Fig. 15.- The NuSTAR 1 X-ray spectrum and best-fitting baseline model are shown in the left panel. The right panel presents the same data together with the best-fitting model including an ionized X-ray wind that accounts well for the deficit of photons in the $6.5-10 \mathrm{keV}$ band (observed frame). 
NuSTAR 2 observation (high radio state) can be interpreted in a similar way. This is unlikely to represent a problem for the claimed wind detection, as the overall statistical result is dominated by the Chandra spectrum, simultaneous with NuSTAR 1. As mentioned, from a radio point of view, the NuSTAR 1 and Chandra observations were performed during a low radio state, while NuSTAR 2 was already in a high radio state. As the NuSTAR 2 spectrum is well described by the baseline model and does not exhibit signs for a deficit of photons in the interesting band, it is tempting to associate the presence of the wind with low radio states.

In order to see whether the peculiarity of the NuSTAR 1 spectrum is indeed well described by an X-ray wind, we add a further ionized absorber to our spectral model for the NuSTAR 1 observation. The additional ionized absorber (modelled via the photoionization code PHASE, see Krongold et al. (2003)) depends on the absorber column density, ionization, and outflow velocity as well as on the gas turbulent velocity. The latter parameter is however fixed to an intermediate value of $500 \mathrm{~km} \mathrm{~s}^{-1}$ as the poor spectral resolution of the NuSTAR data are not sufficient to constrain it.

The ionized absorber provides a statistical improvement of the fit for the NuSTAR 1 data with $\chi^{2}=65$ for 53 degrees of freedom (dof), to be compared with $\chi^{2}=82$ for 56 dof of the baseline model, i.e. the ionized absorber is detected at the 99.4 per cent confidence level according to a simple F-test. The addition of the ionized absorber produces a slightly steeper photon index for the NuSTAR 1 spectrum, which is more in line with that from all others $(\Gamma=1.4 \pm 0.2$ versus $\Gamma=1.2 \pm 0.2$ with the baseline model). All other parameters are the same, within the errors, as those obtained with the baseline model. From our best-fitting model, we infer that the ionized absorber has a column density of $(5 \pm 3) \times 10^{23} \mathrm{~cm}^{-2}$ with ionization parameter $\log U=2.3 \pm 0.3$, and that the gas is outflowing from the system at high velocity, namely $\mathrm{v}_{\text {out }}=17000 \pm 4500 \mathrm{~km} \mathrm{~s}^{-1}$. The NuSTAR 1 spectrum and best-fitting model are shown in the right panel of Figure 15. We note that the wind parameters that we derive from the NuSTAR 1 data are all consistent within the errors with those reported by other authors who made use of a simultaneous Chandra observation as well (Feruglio et al. 2015). This is reassuring in terms of the reality of the wind detection in the NuSTAR 1 observation.

We then perform similar fits including a putative ionized outflow to all other NuSTAR observations, but we do not find any statistical evidence for the presence of such component in any of the high radio state NuSTAR observations (NuSTAR 2-5). This is true also for the NuSTAR 4 observation despite the fact that its spectral shape appears to be the most similar to NuSTAR 1 (see Figure 14), and we only obtain an improvement of $\Delta \chi^{2}=5$ for 3 dof for the NuSTAR 4 observation when a wind model is included. Obviously, this does 
not mean that the wind is absent during the NuSTAR 2-5 observations, but only that its presence is not required by the data (using our specific wind model). The only significant detection is thus obtained during the only low radio flux state observation NuSTAR 1 where it explains well the observed deficit of soft X-ray photons below $12 \mathrm{keV}$. If the flux deficit during the radio weak state is indeed due to an X-ray absorbing wind then this is perhaps suggestive of a phenomenon known to occur in Galactic black hole accreting systems, where jet production is seen to suppress winds of X-ray absorbing gas (Neilsen and Lee 2009). It would also provide an interesting analogy to the suppression of BAL winds in radio loud states noted for Mrk 231 in the last section.

\subsection{The 2015 XMM-Newton observations}

Mrk 231 has been observed three times with XMM-Newton, twice in this observing program and once in 2001. As can be seen from the light curve in Figure 1, the radio state is unknown during the 2001 XMM-Newton observation (Braito et al 2004). So we have two observations during active jet states and one during an unknown epoch of jet activity. Figure 16 shows that there is no measurable difference between the three spectra obtained with XMM-Newton.

Even though the 2015 XMM-Newton spectra are redundant with the 2001 spectrum, we proceed with a simple spectral analysis of these data. XMM-Newton and NuSTAR observed Mrk 231 simultaneously on 2015 May 28. We then start our analysis with this data set, performing simultaneous fits to the low and high energy data from the two missions, to gain insights on the overall broadband spectrum of Mrk231. A constant is introduced to account for any cross-calibration issue between the two missions. The XMM-Newton EPICpn data (XMM 3) are considered in the $0.5-12 \mathrm{keV}$ band, while the NuSTAR 5 data $(\mathrm{A}+\mathrm{B}$ detectors) are used in the $3-30 \mathrm{keV}$ band as above. The soft X-ray emission below $\sim 2 \mathrm{keV}$ is dominated by extended emission (star-formation, photo-ionized gas, etc.) and it can be successfully modeled by means of a two-temperature thermal plasma model plus a power law component representing scattered emission. These two components are thought to originate far away from the central engine, and are thus only absorbed by the Galactic column. As for the $\geq 2 \mathrm{keV}$ spectrum, several models are possible (see e.g. Piconcelli et al. (2013)) and we start here from the simplest possible one, namely an extra absorption component at the redshift of Mrk231 acting on a power law continuum plus Fe line (i.e. the same baseline model that was applied to the NuSTAR data in the previous subsection).

This first attempt provides a good statistical result with $\Delta \chi^{2}=170$ for 134 dof. The temperature of the hot plasmas is $\simeq 0.24 \mathrm{keV}$ and $\simeq 0.89 \mathrm{keV}$, and this component con- 
tributes to the soft $0.5-2 \mathrm{keV}$ band with a total luminosity of $\simeq 2 \times 10^{41} \mathrm{ergs} \mathrm{s}^{-1}$. The soft (scattered) power law has a loosely constrained photon index $\Gamma=1.5 \pm 0.4$ and contributes to the $0.5-2 \mathrm{keV}$ luminosity with an additional $\simeq 2.5 \times 10^{41} \mathrm{ergs} \mathrm{s}^{-1}$. As for the high-energy emission, the X-ray nuclear continuum is well described by a power law with $\Gamma=1.45 \pm 0.15$ with an intrinsic $2-10 \mathrm{keV}(10-30 \mathrm{keV})$ luminosity of $\simeq 3.0 \times 10^{42} \mathrm{ergs} \mathrm{s}^{-1}$ $\left(\simeq 4.2 \times 10^{42} \mathrm{ergs} \mathrm{s}^{-1}\right)$. If the spectral model is extrapolated down to $0.5 \mathrm{keV}$, the total $0.5-$ $30 \mathrm{keV}$ intrinsic luminosity of the $\mathrm{AGN}$ turns out to be $\simeq 8.4 \times 10^{42} \mathrm{ergs} \mathrm{s}^{-1}$. However, as the intrinsic continuum is in fact detected only above a few $\mathrm{keV}$ due to intervening absorption, we caution that this value should be treated with caution as we ignore the true AGN spectrum in the $0.5-2 \mathrm{keV}$ band (where standard AGN often exhibit a soft X-ray excess). The neutral absorber has a column density of $6 \pm 3 \times 10^{22} \mathrm{~cm}^{-2}$, consistent with that derived from the NuSTAR 5 data alone $\left(5 \pm 4 \times 10^{22} \mathrm{~cm}^{-2}\right)$. We detect an Fe emission line at $6.6 \pm 0.1 \mathrm{keV}$ with $\mathrm{EW} \sim 180 \mathrm{eV}$. An emission feature appears to be present around $5.7 \mathrm{keV}$ in the XMM 3 and, with less contrast, NuSTAR 5 spectra. The addition of a further emission line with $\sigma$ fixed at $10 \mathrm{eV}$ results in a statistical significant improvement of $\Delta \chi^{2}=-15$ for 2 dof for a line energy of $5.7 \pm 0.1 \mathrm{keV}$ and an equivalent width of $\sim 190 \mathrm{eV}$. No significant absorption feature that may indicate the presence of an ionized wind is seen in the XMM 3 data, as already noted in the analysis of the NuSTAR 5 data alone.

The previous XMM 2 observation that took place on 2015 April 25 can be described with a very similar model. The only noticeable difference is that the Fe band appears to be simpler than on May 28. A simple Fe emission line at $6.7 \pm 0.1 \mathrm{keV}$ with equivalent width of $\sim 200 \mathrm{eV}$ describes well all the emission, casting some doubts on the reality of the $5.7 \mathrm{keV}$ emission line detected on May 28. The intrinsic continuum photon index is very loosely constrained by the XMM-Newton data alone $(\Gamma=1.45 \pm 0.55)$ and it is absorbed by a column density of $(7 \pm 4) \times 10^{22} \mathrm{~cm}^{-2}$. The resulting best-fitting statistics is $\Delta \chi^{2}=79$ for

87 dof, and no features indicate the presence of a further ionized absorber during the XMM 2 observation.

\subsection{Summary of the X-ray - Radio Connection}

The X-ray spectrum of Mrk 231 has been very constant over all epochs. Because of X-ray absorption of the nuclear emission, the very soft X-ray emission below a few keV is dominated by extended components (star-forming regions, scattering, re-emission by photoionized gas, etc.) and the stability of the X-ray emission is expected. On the other hand, the remarkably constant X-ray flux (and spectral shape) above $10 \mathrm{keV}$ indicates that the intrinsic nuclear emission is not varying on timescales of years. It is curious how the far UV and X-ray spectra 
tend to be very stable, quite unusual for a broad line active galactic nucleus. The only epoch where an elevated flux density above $10 \mathrm{keV}$ was seen was with Suzaku (Piconcelli et al. 2013), where it was explained as a transient uncovering of the X-ray nucleus (no radio sampling is available around the Suzaku epoch).

From a radio point of view, besides the XMM 1 observation that was performed during an unknown radio state, the NuSTAR 1 pointing is the only one that is performed during a low radio flux state, while all others (NuSTAR 2-5 and XMM 2-3) are obtained during high radio flux states. It is interesting to note that the NuSTAR 1 spectrum is precisely the only one that cannot be well described by the simple spectral model used for all other observations. The NuSTAR 1 spectrum presents a deficit of relatively soft X-ray photons (mostly in the 6.5-10 keV band), while it is indistinguishable from all others at higher energies, where the intrinsic nuclear emission dominates. This peculiar spectral shape is best explained by the addition of a further X-ray absorption component in the form of a fast ionized wind with outflow velocity $v_{\text {out }} \sim 17000 \mathrm{~km} \mathrm{~s}^{-1}$. Such a wind solution is not statistically required by the data in any of the high radio state observations. The presence of an ultra-fast Xray wind in August 2012 was claimed previously by others who made additional use of higher quality Chandra data, simultaneous with NuSTAR 1 (Feruglio et al. 2015). The wind parameters that we derive from the NuSTAR 1 data alone are in excellent agreement with those previously derived, which indicates that our detection and modeling are robust.

There being only one low radio state data point, we do not know if the presence of the wind is related to suppressed jet emission, but it is suggestive to note that our findings are in line with the phenomenology of Galactic black hole binaries where winds and jets seem to be mutually exclusive. If the X-ray wind was indeed present (or stronger) only during periods of suppressed jet activity, it would also link well with the suppression of BAL winds during high radio flux states, as noted in the previous section.

\section{Conclusion}

In this article we used our long term radio monitoring at high frequency and a target

of opportunity monitoring of a strong radio flare with VLBA, XMM-Newton and NuSTAR in order to probe the radio jet-accretion disk connection in Mrk 231. 


\subsection{Results}

In spite of some information loss due to a poor beam shape in two of our epochs, we were able to determine a few interesting constraints on the jet-disk connection in this quasar.

1. As discussed in Section 3, two new ejected components in Mrk 231 were detected for the first time (see Figures 4 and 7 )

2. In Section 3.2, we were able to constrain the apparent speed of one of these ejections $>3.15 \mathrm{c}$.

3. In section 3.3, we showed in Figure 8 that the stationary secondary component (the putative radio lobe) doubled in brightness in 9 years.

4. In Section 4, we determined that the far UV emission beyond the peak of the far UV SED was depressed in the two active jet states compared to the one quiescent jet state monitored with HST (see Figure 12 and Table 2).

5. In Section 4, we demonstrated that the degree of CIV broad line absorption in the two active jet states is suppressed compared to the one quiescent jet state monitored with HST (see Figure 12 and Table 2).

6. In Section 5, we found that the $3-12 \mathrm{keV}$ flux is elevated during a state of high radio jet activity relative to the one $3-12 \mathrm{keV}$ flux measurement during the only X-ray observation known to occur in a quiescent radio state (see Figure 14).

\subsection{Physical Interpretation of Results}

The primary result of this VLBI observation program was the detection of ejected components and estimating the apparent velocity, $v_{\text {app }}>3.15 \mathrm{c}$. This allows us to attain new kinematic insight into the jet. Previously, using the methods of Ghosh and Punslv (2007), the time variability brightness temperature is $T_{b}=(12.4 \pm 3.5) \times 10^{12} \mathrm{~K}$ (Reynolds et al. 2009). In order to avoid the inverse Compton catastrophe, the analysis in Ghosh and Punsly (2007) indicates that the line of sight to the jet is less than $\theta_{\max }=\left(25.6^{\circ}\right)_{-2.2^{\circ}}^{+3.2^{\circ}}$. This equates to a time variability factor $\delta>\left(T_{b} / 10^{12} K\right)^{1 / 3}=2.3$ for an optically thick, unresolved core (Ghosh and Punsly 2007). The Doppler factor, $\delta$, is given in terms of $\Gamma$, the Lorentz factor of the outflow; $\beta$, the three velocity of the outflow and the angle of propagation to the line of sight, $\theta ; \delta=1 /[\Gamma(1-\beta \cos \theta)]$ (Lind and Blandford 1985). Figure 17 combines our previous findings with the new estimate of the component apparent velocity, 
$v_{\text {app }} / c=\beta \sin \theta /(1-\beta \cos \theta)$. The figure plots $v_{\text {app }} / c$ as a function of $\delta$. The theoretical plots are made for various lines of sight (LOS) that are allowed kinematically. We now have two constraints on $\delta$ and $v_{\text {app }} / c$ if we assume a steady jet structure over time. The intersection of the two lower bounds is shaded in grey. The arrows indicate the direction of increasing $\Gamma$ on each curve. It is interesting that the new constraint does not significantly improve the previous limit on the LOS, $\theta_{\max } \approx 23.4^{\circ}$. The estimate of $v_{\text {app }} / c$ essentially indicates consistency with the time variability Doppler factor analysis. Combining the two conditions in Figure 17 does indicate that $\Gamma>3.5$ which is a new constraint on the jet kinematics. This highly relativistic motion is consistent with a point of jet origin deep within the gravitational potential well, possibly the innermost regions of the accretion disk.

In principle, polarimetry can provide useful diagnostics of jet activity. Unfortunately, in Reynolds et al. (2009), it was found that there was no detectable polarized flux in our VLBA observations. Presumably, the emission is depolarized by a large scattering column density during the radiative transfer through the enveloping dense nuclear environment. Consequently, the 2015 observations were not a polarization experiment, there was no polarization calibrator in order to maximize the amount of time on the source. Even so, we looked for evidence of polarization and could not find any.

Since, the relativistic jet has a plausible origin in the inner accretion disk there is a straightforward interpretation of point 4, above. A deficit of far UV emission in active jet states suggests a connection to the "EUV deficit" of radio loud quasars: radio loud quasars show a tendency to have a deficit of extreme ultraviolet (EUV) flux shortward of the peak of the SED around $1100 \AA$ relative to the EUV flux levels found in radio quiet quasars (Zheng et al. 1997; Telfer et al. 2002; Punsly 2014, 2015; Punsly et al. 2016). The magnitude of the deficit increases with the strength of the jet (Punsly 2015). The analogy is not direct because the SED of Mrk 231 is extremely unusual and not well understood, especially in the UV (Veilleux. Trippe and Hamann et al. 2013; Veilleux. Melendez and Tripp et al. 2016). The continuum is likely observed through multiple "exotic" absorbing media that differ from the Galactic interstellar medium (Reynolds et al. 2013a; Veilleux, Trippe and Hamann et al. 2013). The far UV flux is highly depressed in overall magnitude, yet the SED is very flat from $1400 \AA$ to $2000 \stackrel{\circ}{A}$, not typical of absorption by a dusty interstellar medium (see Figure 12). However, we can draw one commonality with the EUV deficit of radio loud quasars, the flux is lower at wavelengths shortward of the far UV peak of the SED when there is an active jet as compared to the flux level when the jet is in a quiescent state. It might be that the disk in Mrk 231 is very cool as a consequence of energy losses due to winds driven by the continuum that is emitted from the inner disk (Laor and Davis 2014). Regardless of the reason for the low frequency of the SED turnover, the highest frequency optically thick thermal emission should represent emission from the innermost accretion disk. This emission 
should be detected near or just blueward of the far UV break in the SED (Sun and Malkan 1989; (Szuszkiewicz et al. 1996). Figure 12 seems to indicate that the optically thick thermal emission from the innermost accretion disk (i.e., emission just shortward of the peak of the $\mathrm{SED}$ ) is lower when there is a radio jet similar to the "EUV deficit" in radio loud quasars. In particular, the "EUV deficit in radio loud quasars" has been shown to be consistent with the predicted trends of a simple magnetically arrested accretion scenario in which islands of large scale vertical magnetic flux thread the innermost accretion flow in radio loud quasars. This distribution of flux is located in the base of the relativistic jet and at the same time displace the optically thick gas responsible for the thermal emission from the innermost accretion disk, thereby reducing the flux beyond the peak of the SED relative to that of radio quiet quasars (Punsly 2014, 2015). Applying this dynamic to Mrk 231, the displaced far UV emitting gas equates to a diminished far UV luminosity and the increased vertical flux creates a stronger jet. This creates an inverse correlation between the far UV luminosity and jet power.

The other possibly very interesting finding is point 5 , above. Recall that the existence of large scale jets and BAL winds are almost mutually exclusive. The propensity for suppressed large scale emission increases strongly with BALnicity index (Becker et al. 2000, 2001). Figure 12 and Table 2, indicate that Mrk231 might be a unique source in which we can see the relativistic jet and high ionization BAL wind anti-correlation occurring in real time. This wind suppression is naturally explained by models in which the BAL wind is launched from the innermost regions of the accretion flow, including the outer boundary of the central accretion vortex (Punsly 1999a, b). In these models, the propensity of a quasar accretion flow to launch either a BAL wind or a radio jet is determined by the ratio of radiation pressure to magnetic pressure of the large scale magnetic field in the central vortex and the inner edge of the accretion disk. Thus, points 4 and 5 are consistent if the jet and BAL wind are both launched from the inner accretion disk and accretion vortex. In Mrk231, the ratio of magnetic pressure from magnetic islands and thermal radiation pressure from optically thick gas vacillates. Sometimes the magnetic islands are plentiful and a modest relativistic jet is launched (suppressing the BAL wind and far UV) and at other times the magnetic islands have diffused away and the spectrum of Mrk 231 hardens in the far UV and a high ionization wind is launched.

One might wonder how the increased X-ray flux during the state of high jet activity in point 6 is consistent with these other two findings. The most direct interpretation is that the $\mathrm{X}$-ray excess in the high radio state is a consequence of inverse Compton emission from the same electrons that produce the synchrotron emission in the jet. The spectral index would be slightly steeper than that of the X-ray power law of the coronal X-rays. Considering the superluminal speed in point 2 , above, one would expect significant Doppler enhancement of a 
very weak X-ray source and the resultant flux is likely highly variable. The other possibility is that the reduced X-ray flux in the radio low states is a result of an X-ray absorbing wind (Feruglio et al. 2015). When the jet is active, the dynamics of this X-ray wind launching mechanism (X-ray radiation pressure from the corona) is disrupted by the magnetic islands. The data are not sufficient to distinguish between the two possibilities. However, we show in Section 5, that the difference in the NuSTAR spectrum during the low radio state compared to the high radio states is well described by the presence of absorption in a photo-ionized wind. This is the more intriguing of the two possibilities since it suggests that the existence of a strong jet suppresses both the UV absorbing and the X-ray absorbing winds. The latter is a phenomenon known to occur in Galactic black hole accreting systems, where jet production is seen to suppress X-ray winds (Neilsen and Lee 2009). This could be the first evidence of a direct analogy between the jet-wind connection in supermassive black holes and stellar mass black holes.

\subsection{Future Observations}

Based on these three important inferences of our study, it seems that obtaining one or two HST COS G140L observations in a state of low jet activity would verify if there is a trend for far UV suppression and if the CIV broad absorption trough returns. Also, based on Figure 14, it would be interesting to monitor Mrk 231 in a weak jet state in order to see if the Xray emission decreases. A combined NuSTAR and XMM-Newton (or Chandra) observation

could verify if the X-ray driven wind that was claimed in this low state in Feruglio et al. (2015) returns. Furthermore, longer X-ray observations are required to constrain the Xray driven wind in both the high and low radio sates. If VLBA observations are obtained during another radio flare, it is important to stress the requirement that the $\mathrm{u}-\mathrm{v}$ coverage not be compromised by splitting the observation for scheduling purposes. If the observations are split, care must be taken to ensure that two unique LST ranges are attained on the two consecutive days. It is far better to delay the observation a week or two than compromise the $\mathrm{u}-\mathrm{v}$ coverage. With this constraint, a multi-epoch campaign should see an ejected component evolve in space and time. A HST COS G140L observation in the the rising high flare state has not been obtained and it would be of interest to see if the spectrum is the same as other times during the jet formation. 


\section{Acknowledgments}

This research has made use of data obtained with NuSTAR, a project led by Caltech, funded by NASA and managed by NASA/JPL, and has utilized the NUSTARDAS software package, jointly developed by the ASDC (Italy) and Caltech (USA). We also made use of data obtained with XMM-Newton, an ESA science mission with instruments and contributions directly funded by ESA Member States. This work made use of Director's Discretionary Time on both NuSTAR and XMM-Newton for which we thank Fiona Harrison and Norbert Schartel for approving, as well as the NuSTAR and XMM-Newton SOC for implementing and coordinating the observations. We would like to thank S. Veilleux and M. Melendez for sharing their G130M data. The National Radio Astronomy Observatory is a facility of the National Science Foundation operated under cooperative agreement by Associated Universities, Inc. This research has made use of NASA's Astrophysics Data System Bibliographic Services. This work made use of the Swinburne University of Technology software correlator, developed as part of the Australian Major National Research Facilities Programme and operated under licence.

\section{REFERENCES}

Becker., R. et al. 2000, ApJ, 538, 72

Becker., R. et al. 2000, ApJS, 135, 227

Blandford, R. and Königl, A. 1979, ApJ 23234

Blundell, K., Beasley, A., Bicknell, G. 2003, ApJL, 591, 103

Braito, V. et al 2004, A \& A 42079

Briggs, D., 1995, PhD Thesis, NRAO

Bruni, G. et al. 2013 A\&A 55494.

Brunthaler, A. et al. 2000, A\&A, 357, L45

Deller, A. et al. 2011, PASP, 123, 275

Edelson, R., 1987, ApJ, 313, 651

Evans, I. and Koratkar 2004, ApJS 15073

Feruglio, C.; Fiore, F.; Carniani, S. et al. 2015 A \& A, 58399 
Gallagher, S. et al. 2002 ApJ, 569655

Ghosh, K. and Punsly, B. 2007 ApJL 661139

Ginzburg, V. and Syrovatskii, S. 1969, Annu. Rev. Astron. Astrophys. 7375

Hayashi, T., Doi, A., Nagai, H. 2013 ApJ 7724

Kettenis, M., van Langevelde, H. J., Reynolds, C. \& Cotton, B., 2006, Astron. Data Anal. Software Syst. XV, 351, 497

Kalberla, P.,; Burton, W., Hartmann, D. et al. 2005, A\&A, 440, 775

Klein-Wolt, M. et al., 2002, MNRAS 331745

Knigge, C., Scaringi, S., Goad, M., Cottis, C. 2008 MNRAS 3861426

Krongold, Y., Nicastro, F., Brickhouse, N. S., Elvis, M, Liedahl, D., Mathur, S. 2003 ApJ, 597,832

Laor, A., Davis, S. 2014 ApJ 4283024

Lind, K., Blandford, R. 1985, ApJ 295358

Lipari, S., Colina, L., Macchetto, F. 1994, ApJ 427174

Macleod, C. 2012, ApJ 753, 106

McCutcheon, W., Gregory, P. 1978, AJ, 83, 566

Moffet, A. 1975 in Stars and Stellar Systems, IX: Galaxies and the Universe, eds. A. Sandage, M. Sandage \& J. Kristan (Chicago University Press, Chicago), 211.

Neilsen, J. and Lee, J. 20o9, Nature, 458, 481

Piconcelli, E., Miniutti, G., Ranalli, P. et al. 2013, MNRAS, 428. 1185

Prat, L., Rodriguez, J, and Pooley, G. 2010, ApJ 7171222

Punsly, B. 1999a, ApJ 527609

Punsly, B. 1999b, ApJ 527624

Punsly, B. 2006, ApJ, 647, 886

Punsly, B. 2014 ApJL 79733 
Punsly, B. 2015 ApJ 80647

Punsly, B., Marziani, P., Kharb, P., O’Dea, C., Vestergaard, M.. 2016 ApJ 81279

Reynolds, C., Punsly, B. Kharb, P., O’Dea, C. and Wrobel, J. 2009, ApJ, 706, 851

Reynolds, C., Punsly, B. and O’Dea, C. P. 2013a, ApJL, 773, 10

Reynolds, C., Punsly, B., O’Dea, C. P., and Hurley-Walker, N. 2013b, ApJL, 776, 21

Shankar, F., Dai, X., Sivakoff, G., 2008 ApJ 687859

Sun, W.-H., and Malkan, M. A 1989, ApJ 34668

Szuszkiewicz, E., Malkan, A., and Abramowicz, M. A. 1996, ApJ 458474

Teng, S. H., Brandt, W.N., Harrison, F. A. et al. 2014, ApJ, 758, 19

Telfer, R., Zheng, W., Kriss, G., Davidsen, A. 2002 ApJ 565773

Tucker, W. 1975, Radiation Processes in Astrophysics (MIT Press, Cambridge).

Ulvestad, J., Wrobel, J. and Carilli, C. 1999, ApJ, 516, 134

Ulvestad, J. et al. 1999, ApJL, 517, L81

Veilleux, S., Trippe, M., Hamann, F., et al. 2013, ApJ, 764, 15

Veilleux, S., Melendez, M., Tripp, T., Hamann, F., and Rupke, D. 2016, ApJ, in press

Welsh, B., Wheatley, J., and Neil, J. 2011, A \& A 527 A15

Wilhite, B. et al. 2005, ApJ 633638

Weymann, R.J., Morris, S.L., Foltz, C.B., Hewett, P.C. 1991, ApJ, 373, 23

Weymann, R. 1997 in ASP Conf. Ser. 128, Mass Ejection from Active Nuclei ed, N.Arav, I. Shlosman and R.J. Weymann (San Francisco: ASP), 3

Willott, C., Rawlings, S., Blundell, K., Lacy, M. 1999, MNRAS 3091017

Zhang, S. et al. 2010, ApJ, 714, 367

Zheng, W. et al. 1997 ApJ 475469

Zwart, J. et al. 2008, MNRAS, 391, 1545

This preprint was prepared with the AAS IATEX macros v5.2. 


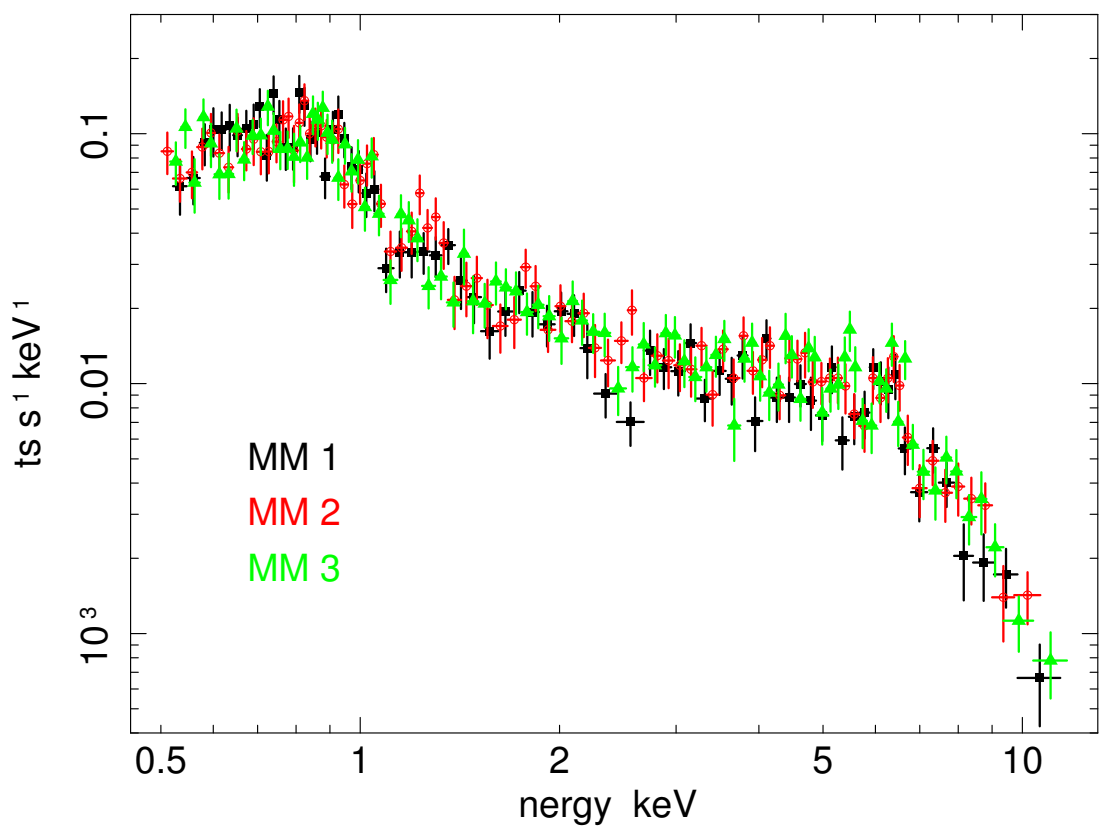

Fig. 16. - The EPIC-pn spectrum from the three epochs of XMM-Newton observations show no significant spectral/flux variability. 


\section{Apparent Velocity vs. Doppler Enhancement}

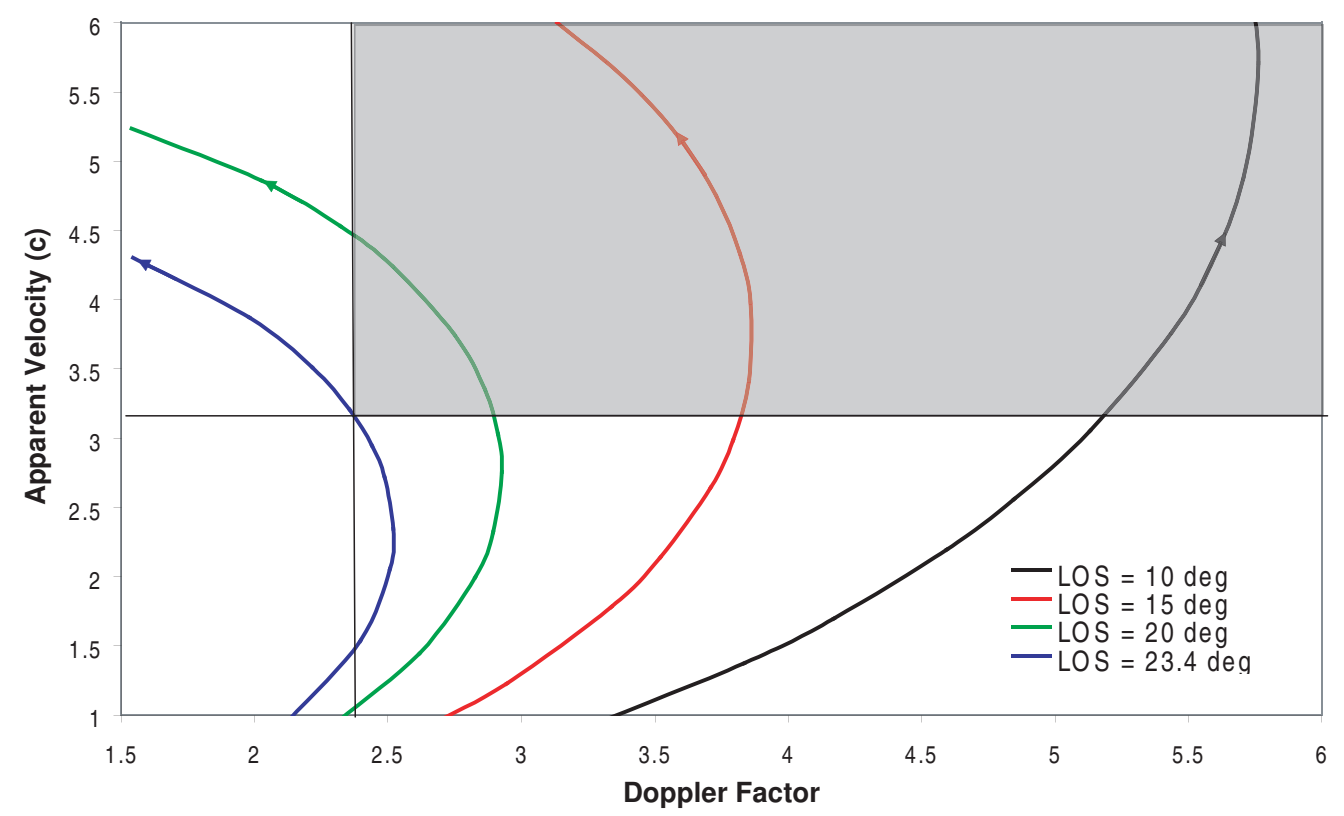

Fig. 17.- The apparent velocity versus Doppler factor for various lines of sight (LOS). The shaded grey area is consistent with the lower bounds on the time variability Doppler factor from Reynolds et al. (2009) and $v_{\text {app }}$ from this paper. The arrows indicate the direction of increasing $\Gamma$ along each curve. 